\title{
MASTER
}

\section{TFTR MIRNOV COIL ANALYSIS AT PLASMA START-UP}

Thomas R. Harley, Dean A. Buchenauer, John Coonrod, Kevin M. McGuire

\author{
Plasma Physics Laboratory, \\ Prisceton University, \\ PPPL- 2298 \\ Prince:on, New Jersey 08.544 \\ DE86 006354
}

ABSTRACT. Tle methods for finding poloidal and toroidal numbers of MHD oscillation: from Mirnov coils are reviewed and modifed. Examples of various $\mathrm{MHD}$ phenomena occurring during start-up on TFTR are illustrated. It is found that the MHD mode structure best fits a model with the torojdal correction ${ }^{1,2}$ included.

A sew algorithin which finds $m, n$ numbers can accommodate toroidal effects which are manifested in the phase data. The algorithm can find $m, n$ numbers with a given toroidal correction parameter $\lambda^{\prime},\left(\lambda^{\prime}=0 \Rightarrow c y\right.$ lindrical $)$. This algorithm is also used to find the optimal value of $\lambda^{\prime}$ automatirally, eliminating the need for "guesswork." The algorithm finds the best parameters to the fit much faster than more conventional computational techniques.

\section{DISCLAIMER}

\begin{abstract}
This report was preparod as an account of work sponsored by an agency of the United Slates Government. Neither the United States Government not any agenty thereof, not any of their employes, makes any warranty, express or implied, or assumes any iegal liability or responsibility for the accuracy, completeacss, or use[u]nces of any information, apparalus, produch, or process diselood, or represents that its use would not infringe privately owned rights. Reterepre hercin to any spocitic commercial product, process, or service by trade name, trademark, manufacturer, or otherwise does not necessarily constitute or imply its endorsement, rocommendation, or favoring by the United States Government or any agency thereof. The views and opiations of authors expessed hetein do not necessarily staic or reflect those of the L'nited States covernment or any Jgency thereof.
\end{abstract}




\section{INTRODUCTION}

Evidence of magnetic islands in a tokamak plasma is given by the external magnetic field fluctuations which Mirnov coils detect. Correlating the phases from an array of Mirnov coils yields useful information about the geometry of a mode's magnetic perturbations. In Sec. Il of this paper, the techniques involved in analyzing data from an array of Mirnov coils are reviewed and modified. This data analysis takes into account the increased spatial variation of the mode on the inner side of the tokamak. The analysis techniques are especially helpful for analyzing modes in the plasma with $m \geq 4$.

Section IIİ contains results obtained by using these analysis techniques on TFTR startup data. Particular emphasis is given to measuring the degree of poloidal asymmetry in the magnetic perturbations. These results are compared with theoretical predictions for the first order toroidal correction. Efects due to the displacement of the node's center away from the vacuum vessel center are also investigated. The combined effect due to toroidicity and the off-center position provides a strong motivation for using a free torridal correction parameter when fitting the phase data from the Mirnov coils.

An algorithm was developed to mirimize the computational time and length of code necessary to calculate the m.n numbers for a given sel of phase data. This algorithm is particulariy useful for anyone analyzing Mirnow cojl data in order to find $m, n$ numbers. Analytical solutions for the parameters in the phase fit may be found for all the parameters used in the fit to the Mirnov phase data.

\section{METHOD}

Tae locations of the Mirnov coils in TFTR are given in Fig. 1. The coils are located $110 \mathrm{~cm}$ from the vacuum vessel center and are oriented in order to detect $\dot{\vec{B}}_{\theta}$. The poloida] array forned by coils 1-16 detect the $m$ number and coils $17-19$ are used to determine the $n$ number. Techniques for analyzing data from these signals are well known. First a Mirnov signal, depicted in Fig. 2(a), is inspected for regions of high WHD activity. After expanding the region of interest, the $\dot{\bar{B}}_{\theta}$ signal usually appears sincsoidal when a clear mode is present. If the signal is iarge enough, une can often determine the $m$ number of the mode as the number of maxima that pass by at at fixed time on an overlaid plot of the Mirnov signals from the poloidal array. as Fig. 2(b) illustrates. This method is less misleading if the signal from the first coil (plotted at the bottom) is plotted again at the top ?unlike the diagram in Fig. 2(b) to more easily see that the signal on channel 1 is "lagging" far behind channel 16 rather than "leading" it slightly, so that 6 "ridges" can be observed to cross any vertical line. 
One should choose a time range which is large enough for a fast Fourier transform to single out relevant oscillation frequencies in the signal above the background Ioise. The time range should be small enough, however, so that the mode's frequency does not vary too much over the time range to be fast Fourier transformed, and so that regions where too much noise is on the signals may be avoided. Time ranges for which a mode was present and the data were suitable for phase correlation analysis were most efficienily found by observing Mirnov signais from the poloidal array on overlaid plots.

Analysis of the data shown in Fig. 2(b) is given in Fig. 3. Although the average of all the Mirnov coil's amplitudes at a given frequency for a wide range of frequencies [Fig. 3(a)] has little quantitative meaning, it is useful to determine the frequency of the mode which the Mirnov coils detect. The corresponding data from a single Mirnov coil may contain misleading peaks due to noise on that signal.

Having chosen the frequency of interest, the phase angles from each Mirnov coil taken at that frequency are investigated as in Fig. $3(b) \mid$. At this point one may attempt to find the best cyitindrical fit 'Fig. 3 (c)] to the phases. This involves finding the optimal $m, n, \delta_{i}$ parameters such that $\xi=m \theta+n \varphi+\delta_{i}$, approximates the phase data. Here $\delta_{i j}$ is a phase cunst ant and $\theta$. $\phi$ are the poloidal and toroidal angles of the Mirnov coils. A more reliable method for finding $m, n$ numbers (especially ior $m \geq 4$ ) is finding the optimal fit with four free parameters, i.e.. $\xi=m\left(\theta+\lambda^{\prime} \sin \theta\right)+n \phi+\delta_{11}$. The added free (Loroidal correction) parameter, $\lambda^{\prime}$, enables the fit to allow for toroidal corrections to the geometry of the mode and the off-center position of the mode with respect 10 the center of the vacuum ressel. Figure $3(\mathrm{~d})$ illustrates the optimal toroidal fit.

Figure 4 jllustrates typical failure of the cylindrical approximation to determine $m . n$ numbers corsectly. The optimal cylindrical fit is depicted by the dashed line whirh indicates $m=3$. The solid ine indicates the contrasting optimal toroidal fit which determined $m=4$ correctly. This solid line fit was found by leaving $\lambda^{\prime}$ as a free parameter. The number of times the fit wraps around the top of the graph indicates the mumber of the fit.

Since $\lambda^{\prime}$ is found by the cumputer, the difficulty of having to estimate $\lambda^{\prime}$ for the flux surface on which the mode lies is removed. This zutomation is especially useful for analyzing starı-up data ( $m \geq 4)$ to obtain $m, n$ numbers as well as gain useful information about the geometry of the tearing mode:

Another method is available for obtaining a more concrete idea of what the geometry of the mode's poloidal field variations looks like. ${ }^{4}$ Let $a(i)$ and $p h(i)$ be the amplitude and phase angle for each Mirnov coil taken at the fraquency of interest. Let $\theta(i)$ represent the poloidal angle of the $i^{t h}$ ilimov coil. Define $\dot{r}(\theta)$ as the cubic spline fit which interpolates 
the data points, $\{\theta(i), r(i)=a(i) \cos [p h(i)]+C\}_{i=1}^{N}$. A polar plat of the $\hat{r}(\theta)$ fit then indicates the $m$ number, according to the number of "humps" that are present. These polar plots can be thought of as a "snapshot" of the mode's poloidal magnetic field variations. Emphasizing only one Fourier component from the signals merely attempts to filter out noise from sources other than the mode.

This method breaks down for large values of $m$. As the difference between the phases from adjacent Mirnov coils (1-16) increases, it becomes more probable that one of the "humps" will be missed. Figure 5 illustrates four different methods of analyzing the same data. In Fig. 5(a) the optimal cylindrical fit indicates $m=4$, and in Fig. 5(b) the polar plot indicates $m=5$. The optimal toroidal fit shown in Fig. 5(c) correctly determined that $m=$ 6. A careful examination of a corrected $m=6$ polar plot in Fig. 5 (d) reveals that a "hump" between the $16^{\text {th }}$ and $1^{\text {th }}$ Mirnov coils was missed in Fig. $\mathrm{s}(\mathrm{b})$. Figure 5 (b) demonstrates that the $m$ number resolution obtained from directly inkerpolating the $\{\theta(i), r(i)\}_{i=1}^{N}$ data was unreliable for $m>5$. The technique that was used to produce the corrected polar plot in Fig. 5(d) was accurate for much higher m numbers.

The technique employed to construct this corrected $m=6$ polar plot is illustrated in Fig. 6. The (optimal torojdal) phase fit is depicted by the curved solid line in Fig. 6(a). The difierences between the Mirnov coil phases and this phase fit are evaluated at each Mirnor roil position and plotted against poloidal angle in Fig. 6(b). This is interpolated by a cubic spline, which is also given in Fig. 6(b). This spline is added to the phase fit in order to obtain a corterled phase fit which actually interpolates the phase data. The cubic spline fit in Fig. 6(c) interpolates the amplitude data of the Mirnov coils (versus poloidal angle). Sow 1 he $\left\{\theta(i) . r(i)=a(i) \cos _{1} \theta(i)^{\circ}+C\right\}_{i=1}^{N}$ data are interpolated with the product of the amplitude spline times the cosine of the corrected phase fit (plus a constant) in Fig. 6(d). To reiterate this mose symbolically, let the interpolating cubic spline (ics) of $\left\{x_{z}, y_{i}\right\}_{z=1}^{N}$ be denoted by $y(x)=i c s(x)=i c s \mid\left\{x_{i}, y_{t}\right\}_{i=1}^{N}, x^{\prime}$. The fit, $\xi(\theta)=m\left(\theta+\lambda^{\prime} \sin \theta\right)+n \phi+\delta_{0,}$ is adjusied to $\hat{\xi}(\theta)=\xi(\theta)+i c s\left[\{\theta(i), p h(i)-\xi \theta(i) \mid\}_{i=1}^{N} . \theta_{i}^{i}\right.$ so that $\hat{\xi}^{\prime} \theta(i) !=p h(i)$. Letting $\dot{a}(\theta)=i c s i\{\theta(i), a(i)\}_{i=1}^{N}, \theta \mid$, the corrected polar plot is then constructed from $\vec{r}(\theta)=$ $\dot{a}(\theta) \cos i \xi(\theta) i+C$. This technique gives a much better picture of the mode's geometry as Fig. 6(d) illustrates. Using the interpolation of the phase data, ph(i), instead of only utilizing the rosine of the phase data by directly interpolating $r(i)=a(i) \cos p h(i)-C$. avoids losing useful information about the phases, for example, $\cos \left(-15^{\circ}\right)=\cos \left(15^{\circ}\right)$.

The polar plot in Fig. 6(e) depicts the same data and interpolation as Fig. 6(d): plotted against $\theta^{*}=\theta+\lambda^{\prime} \sin \theta$ instead of $\theta$. This should transform the mode into a cylindrical geometsy, according to the degree that $\xi=m \theta^{-}-n \dot{\theta}+\delta_{1}$, is an appropriat. 
model for the phase data.

All of the polar plots given in the remainder of this paper show $\vec{r}(\theta)$ plotted against $\theta$ as obtained by the described method for constructing corrected polar plots.

\section{PHYSICS}

With the exception of Fig. 19 all of the data shown in this report was taken from shot \#9689 (6/22/84) during start-up. This was a typical discharge for TFTR. The current flat topped at $1 \mathrm{MA}$ at $t=1 \mathrm{sec}$. This was an ohmic heating discharge with $B_{T}=2.8 \mathrm{~T}$.

\section{A. Parameters}

Figures $7-8$ give the relevant parameters of the shot for the 0 to $0.6 \mathrm{sec}$ time range for which aralysis from the Mirnov coils will be given. $t=\beta_{\theta}-l_{1} / 2$ (where $l_{1}$ is the internal incuctance per unit length), the major radius of the plasma, the plasma cursent. and the Shafranov $q$ value at the edge of the plasma are shown in Fig. $i$. Figure 8 gives the line-averaged density.

H. Evolution of detected modes

Figure 9 gives the Mirnov signal on coil 9 (see Fig. 1). The modes which were detected and the time range for which they could be found is shown above the signal. A corselation between increased MHD activity and the time ranges when a mode is present can easily be ohserved.

Figures 10-11 illustrate the $\mathbf{i}$ different modes that were detected in their chronological order. Fote that the phase data and corresponding phase fits typically show greater spatial variation on the inner sjde of the tokamak (near $\theta=0^{\circ}$ or $\theta=360^{\circ}$ ). Talle 1 gives the 1jme range which was Fourier analyzed, frequency, Shafranov $q$ value at the edge of 1 he plasma. $\delta B_{\mathrm{E}} / B_{\mathrm{H}}$, and $\lambda, \vec{j}$, for $i$ modes. To within the experimental accuracy of $q_{*}(a)$, it was found that $m / n \leq q_{*}(a)$. Since $n=1$ for all 7 modes, the $m$ numbers generally decreased as ge (a) decreased with time.

Figures 1213 show the same 7 modes on polar plots. The inner sjde of the tokamak is to the right of each of the polar plots. The typically increased spatial variation of the mode on the inner side can easily be observed. In Fin. 1.t the phases have becn negaled. shifted relative to the phase of Mirnox coil $\neq 1$, and added 10 appropriate multiples of $360^{-}$in ordes to display the phase data from the $i$ modes in a more continuous manner. Alt hough $\lambda^{\prime}$ is not the same for the 7 modes, a rough $m \sin \theta$ dependence in the toroidal 
effect can be seen in Fig. 14. This agrees with the $\xi=m \theta+\lambda^{\prime}(m \sin \theta)+n \phi+\delta_{0}$ model.

\section{Minor Disruptions}

Figure 15 depicts two minor disruptions. The first disfuption occurred at the end of an $m=8, n=1$ mode which was quickly followed by an $m=7, n=1$ mode after a negative loop voltage spike was observed. Later the $m=i, n=1$ mode grew very large and disrupted, but remained after the negative loop voltage spike. Figure 16 shows $l_{p}, R_{p}, q_{s}(a)$, and $A=\beta_{\theta}+l_{i} / 2$ data over the time range in which the two minor disruptions occurred. The growth rates for the first and second modes preceding the two disruptions were estimated from the integrated Mirnov signal to be $900 \mathrm{sec}^{-1}$ and $300 \mathrm{sec}^{-1}$, respectively. Rough estimates of the Alfvén and resistive time scales of the plasma were made using $R_{\mathrm{rnn}}=255 \mathrm{~cm}, a=80 \mathrm{~cm}, n_{e}=4 \cdot 10^{12} \mathrm{~cm}^{-3}, B_{T}=2.8 T$. and $v_{l o n}=3.5 \mathrm{~V}$. For these parameiers $\tau_{A}=0.084 \cdot 10^{-0} \mathrm{sec}$ and $r_{R}=6.5$ sec. giving a magnetic Reynold's number of $S=\tau_{R} / \tau_{A}=7.7 \cdot 10^{7}$. Assuming the current profile was such that these modes were unstable, the linear theory ${ }^{5}$ predicts a growth reie of $i=\alpha \tau_{A}^{-2 / 5} \tau_{R}^{-3 / 5}$. Although the growth rate coefficient $\alpha$ depends on the current profile. which is not known, $\alpha$ is on the order of unity and $\tau_{A}^{-2 / \bar{s}} \tau_{R}^{-3 / 5}=220 \mathrm{sec}^{-1}$.

\section{Coupled Modes}

Figure 17 shows the Mirnov signal from coil 49 and its Fourier 1 ransform. The time range over which the fast Fourier transform was performed on the Mirnov fata was from 5.35-550 m.s as is indicated on the graph. Simultaneously existing modes were found with $m=3, n=1$ and $m=4, n=1$ at 1.0 and $0.8 \mathrm{kHz}$, respertively, as indicated on the Fourier transform graph in Fig. 17. Perhaps the relative weakness of the $m=3$ modes signal reflects the fact that the $m=3$ mode lies deeper in the plasma and further away from the Mirnov coils than the $m=4$ mode. Figure 18 illustrates a superposition of the polar plots from the coupled modes.

\section{E. Electron Diamagnetic Drift Direction}

Figure 19 demonstrates two modes rotating in the electron diamagnetir drift direction ( $"$, the plasma current and toroidal magnetic field reversed, so $x^{\prime},{ }^{\text {th }}$ is in the opposite direction. Metion of the mode is essential for Mirnov coils to detect il. 


\section{F. Toroidal effect on mode geometry}

1. Experiment

A measure of the relative accuracy by which a phase fit approximates the $N$ phase data points is given by the reduced ch:-squared statistic, ${ }^{3,7}$ denoted $\chi^{2}$. Lower $\chi^{2}$ values indicate more accurate fits. Minimizing $\chi^{2}$ is the basis by which the phase parameters $m, n, \delta_{0}$ and optionally $\lambda^{\prime}$ are chosen. The code to implement this minimization actually evaluates $\mathrm{CHI}$, where $\chi^{2}=\mathrm{CHI} 2 * 3 /\left[(\mathrm{N}-\nu) \pi^{2}\right]$, and $\nu$ is the number of free parameters in the fit. CHI2 is explicitly defined in Sec. IV.

For the modes which were analyzed in Table 2 it was found that the cylindrical fits $\left(\lambda^{\prime}=0\right)$ approximated the Mirnov phase data poorly. The $m, n$ numbers chosen on the basis of optimally misimizing $\chi_{c y l}^{2}$ were inaccurate for all cases in Table 2 . Adding the free parameter $\lambda^{\prime}$ resulted in better fits as indicated by the $\chi_{\lambda^{\prime}}^{2}$ column in Table 2. The optimal choice of $m, n, \delta_{0}, \lambda^{\prime}$ to minimize $\chi \hat{\lambda}^{2}$ determined the corsect $m, n$ numbers of the mode in all cases. Values of $\chi^{2}$, are also given in Table 1, which shows data for the 7 different mades in Figs. 10-14.

Assuming that the modo lies near the outermost (in ninor radius) Hux surface $\left(a_{p}=\right.$ $83 \mathrm{~cm}, r_{m n} \approx 80 \mathrm{~cm}, R_{\mathrm{c}}-265 \mathrm{~cm}$ ), then $R_{n n} \approx R_{1}=255 \mathrm{~cm}$ and one can transform the poloidal angles of the Mirnov coils relative to the vacuum vessel center into poloidal angles relative to the center of the tearing mode (see Fig. 20). $R_{\text {: }}$ and $R_{p}$ are the major radii of the centes of the tohamak vessel and the center of the outermost most flux surface, respectively. This transformation usually improves the accuracy of the subsequently computed toroidal phase fit, as shown under the column headed $x_{\lambda_{m, 1}^{2}}^{2}$ in Table 2. This improvement reflects the fact that the effect of the mode"s off-center position on the phase data can be more accurately accomnodated by a term proportional to $\sin (\theta)$ in the coordinates of the mode. The value of the toroidal corsection parameter for the fit using transformed coordinates is given under the column headed $\lambda_{\text {mode }}^{\prime}$. This $\lambda_{\text {mode }}^{\prime}$ value represents the toroidal correction parameter for coordinates relative to the center of the mode if the mode lies near the outermost flux surface.

Again assuming the mode lies nez: the outermost flux sufface, theory ${ }^{2}$ predicts that $\lambda=\frac{r_{w+1}}{\beta_{m, 1}}(\Lambda-1)$ for $\Lambda=\beta_{\theta}+l_{i} / 2$ evaluated at the edge of the plasma, and $\lambda_{\text {mode }}^{\prime} \approx \lambda-\frac{y}{m}$. (The $\frac{y}{m}$ term will be explained in the following section on theory.) Thus the $\lambda_{\text {mode }}^{\prime}$ values are expected to be equal to $\lambda_{\text {hern: }}^{\prime}=\frac{r_{u+1}}{R_{u+m}}(\Lambda+1)+\frac{y}{m}$ when the mode lies near the outermost fux surface. Here values for $\Lambda$ are measured from the moments of the exiernal magnetic field. Apparently, from the tabulated data given in Table 2, as $y_{3}(a)$ decreases. 
the mode is pushed outward in minor radius towards the outermost fux surface so that the assumptions based on the mode's outward position becorse more valid and $\lambda_{\text {mode }}^{\prime} \rightarrow \lambda_{\text {Mercz }}^{\prime}$ for each of the three modes.

\section{Theory of Tearing Mode Phase Moduiation}

The poloidal magretic field perturbations produced by tearing modes were observed to vary in space more rapidly on the inner side of the lokamak $(\theta=0)$. The phase asymmetry is understood to be primarily due to two effects; toroidicity and an off-center position.

In the infinite aspect ratio cylindrical approximation of a tearing mode, a cursent perturbation of the form $\hat{j}=j_{m n} \cos \left(m \theta+n \phi+\delta_{n}-\omega t\right) \delta\left(r-r_{m n}\right)$, produces variations in the poloidal magnetic field given by ${ }^{3} \tilde{B}_{\mathrm{r}}=-A \sin \hat{\xi}$ and $\tilde{B}_{\mathrm{f}}=A \cos \tilde{\xi}$ where

$$
A=\frac{\mu_{11} j_{m n} T_{m n n}}{2}\left(\frac{r_{m n}}{r}\right)^{m+1} \quad, \bar{\xi}=m n \theta+n \phi+\delta_{01}-\omega t
$$

The toroidal geometry induces a correction ${ }^{3-3,6,7}$ to the mode such that

$$
\bar{\xi}=m \theta+n \phi+\delta_{i-1}-\omega t
$$

with the "Merezbkin" corrertion: $\theta^{\circ}=\theta+\lambda \sin \theta$

$$
\lambda=\lambda\left(r_{m n}\right)=\frac{r_{m n}}{R_{m n}} ; \beta_{H}\left(r_{m n}\right)+\frac{3}{2} l_{\imath}\left(r_{m n}\right)+1 \mid .
$$

$R_{m n}$ and $r_{m n}$ are the minor and major radii of the $m, n$ fux surface, $\beta_{\theta}=\frac{\partial_{\pi}}{\beta_{11}^{2}}\left(\langle p\rangle_{r_{m, n}}-p_{a l}\right)$.

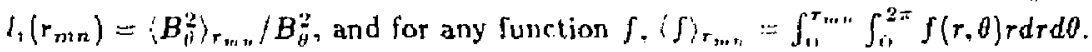

A secondary effect is due to the fact that the center of the mode and the center of the vacuum vessel do not necessarily have the same major radius. ${ }^{6}$ Let the Grad-Shafranov shife' be taken with respect to the outermost, flux surface so that $\Delta(r=a)=0$. Let $s=R_{c}-R_{p}$ so that $d=d(r)=s-\Delta(r)$ gives the exact distance by which the flux starface having minor radius $r$ is off-center with respect to the Mirnov coil array. The $\Delta(r)$ term will subsequently be neglected. Figure 20 illustrates the displacement of the outeimost flux surface and istroduces the variables used to analyze this effect. The distance from the vacuum center to the Mimov coils is $b=110 \mathrm{~cm}$. Letting $y=d / b$ one finds that $\gamma=\arcsin \left[\frac{y \sin A}{\sqrt{1-2 y \cos \theta-t^{2}}}\right]$ and $\theta^{\text {mode }}=\theta+\gamma$ so that $\theta^{-}=\theta^{\text {mode }}+\lambda \sin \theta^{\text {mode }}=$ $\theta-\eta-\lambda \sin (\theta+y)$.

For a typical TFTR plasma $d=10 \mathrm{~cm}(265 \mathrm{~cm}-255 \mathrm{~cm})$ so $y=1 / 11$. For TFTR. typically $\Lambda \leq 1$ so that $\lambda \leq \frac{\frac{b}{2}}{255}(\Lambda+1)<\frac{2}{3}$. Becalise the mode is off-centered, the position 
of each Mimov coil is an angle of $\gamma$ away irom the orientation which would only measure $\tilde{B}_{\theta}$ of the mode. In the low frequency limit, the Mirnov coils measure

$$
\dot{\tilde{B}}=\dot{\tilde{B}}_{\theta} \cos \gamma+\dot{B}_{\mathrm{r}} \sin \gamma=\omega A(\sin \dot{\xi} \cos \gamma+\cos \tilde{\xi} \sin \gamma)=\omega A \sin (\xi), \xi=\dot{\xi}+\eta \quad .
$$

Fourier analyzing each coil's signal in time gives a phase angle

$$
\begin{gathered}
\xi=m\left[\theta+\operatorname{arcsis]}\left[\frac{y \sin \theta}{\sqrt{1-2 y \cos \theta+y^{2}}}\right]+\lambda \sin \left(\theta+\arcsin \left[\frac{y \sin \theta}{\sqrt{1-2 y \cos \theta+y^{2}}}\right]\right)\right] \\
+\arcsin \left[\frac{y \sin \theta}{\sqrt{1-2 y \cos \theta+y^{2}}} j \div n \phi+\delta_{0}-\omega t\right. \\
\vdots=m\left[\theta+\left(\lambda+y+\frac{y}{m}\right) \sin \theta\right]+n \phi+\delta_{0}-\omega t+m \lambda y \cos \theta \sin \theta+O\left(y^{2}\right)
\end{gathered}
$$

This analysis indicates $\lambda^{\prime} \approx \lambda+y+\frac{y}{m}$. The same calculation in mode coordinates, using $\gamma=\arcsin \left(y \sin \theta^{\text {mode }}\right)$, yields

$$
\xi=m\left[\theta^{\text {mode }}+\left(\lambda+\frac{y}{m}\right) \sin \theta^{\text {mode }}\right]+n \phi+\delta_{0}-\omega t+O\left(y^{3}\right), \lambda_{\text {mode }}^{\prime} \approx \lambda+\frac{y}{m} \quad .
$$

Comparison of the phase formulas for physical and mode coordinates reveals that one would expect to obtain lower $x^{2}$ values by converting the physical coordinates into mode conrdinales, since this eliminates the $m \lambda y \cos \theta \sin \theta$ term which the fit parameters cannot accommodate. Transforming to mode coordinates reduces the theoretical errors in the fit from $O(y)$ t.o $O\left(y^{2}\right)$. This improvement in the fit "vas previously verified in the experimental results. It is due to this reduced error that theoretical predictions of the toroidal effect were compared with $\lambda_{\text {mode }}^{\prime}$ rather than $\lambda^{\prime}$.

The phase angle formula for physical coordinates helps to explain why correct. $m, n$ numbers may be obtained by modelling the fit with a free toroidal correction parameter, $\lambda^{\prime}$, without having to "worry" about the off-center position of the mode. First of all, the of-center position of the mode typically produces a smaller correction than the dominant Merezhkin correction, i.e., $y+\frac{y}{m}<\lambda$. Secondly, the effect of an off-centered position cas be approximately accommodated by a term proportional to $\sin \theta$. (The ratio of the maximum effect on the phase fit from the $m \lambda y \sin \theta \cos \theta$ ierm to the maximum effect of 1.he terms due to the ofi-center position which are proportional to $\sin \theta$ is $\left.\frac{1}{2} \frac{m}{m+1} \lambda<1,3\right)$. Thus, the sum of the two effects is allowed for by the free $\lambda^{\prime}$ parameter. The tedious computational task of guessing at the effects of toroidjcity and the off-centered position on the phase data is consequently unnecessary for analyzing typical TFTR data to obtain correct $m, n$ numbers. 
Figure 21 illustrates that for shot \#9689 (when the outermost flux surface was displaced from the vacuum center by $10 \mathrm{~cm}$ towards the center of the tokamak) the Merezhkin correction dominated the effect of the mode's off-center position. Since $q_{s}(a)=6.0$ was measured hey, it is assumed that the $m=6, n=1$ mode is near the edge of the plasma. In Fig. 21 the curved solid line shows the combined effect of the off-center position and the Merezhkin correction. The curved dashed line shows the same radial data plotted against the poloidal angle of the Mirnov coils relative to the center of the mode. Thus the curved dashed line attempts to isolate the effect of the Merezhkin correction from the effect of being off-center (neglecting the $\frac{y}{m}$ term since $\frac{y}{m}<y$ ). This illustrates that for typical TFTR data, the more rapid spatial variation of Mirnov coil phases on the inner side of the tokarnak is predominantly due to toroidicity (or the mode's noncylindrical geometry) rather than being caused by the effect of the mode being off-center relative to the array of Mirnov coils. Note for $R_{p}<R_{c}$ the two effects enhance each other.

\section{ALGORJTHM}

The problem of solving for $m, n$ numbers from the Fourier transform phase angles of $N$ Mirnov coil signals taken at a specific frequency is now defined. Let $p h(i)$ denote the phase of the $i^{\text {th }}$ Mirnov coil (where $-\pi<p h(i) \leq \pi$ ). Let $\theta(i)$ and $\phi(i)$ represent the poloidal and toroidal angles giving the position of the $i^{\text {th }}$ Mirnov coil relative to the tokamak. The parameters $m, n, \lambda^{\prime}, \delta_{0}$ are chosen such that the data, $\{p h(i)\}_{i=1}^{N}$, are best approximated by the fit, $\left\{m\left[\theta(i)+\lambda^{\prime} \sin \theta(i)\right\}+n \phi(i)+\delta_{\mathrm{II}}\right\}_{i=1}^{N}$.

Assume that $m, n, \lambda^{\prime}$ are given and one is trying to find the optimal $\delta_{i j}$. $\delta_{0}$ is determired by minimizing CHI2, the unnormalized chi-squared value of the fit. ${ }^{8} \mathrm{CHI} 2$ is defined in the following heuristic code, in which $\left.\{y(i)\}_{i=1}^{N}=\left\{m \mid \theta(i)+\lambda^{\prime} \sin \theta(i)\right\} \cdot n \phi(i)\right\}_{i=1}^{N}$.

$$
\begin{aligned}
\text { FUNCTION CHI2 }\left(y, p h, N, \delta_{0}\right) \\
\text { CHI2=0. } \\
\text { DO } 10 i=1, N \\
\text { D=ABS }\left(p h(i)-\left(y(i)+\delta_{1}\right)\right) \\
\text { D }=\text { AMOD }(\mathrm{D}+\pi, 2 \pi)-\pi \\
10 \quad \text { CHI2=CHI2+D*D } \\
\text { RETURN }
\end{aligned}
$$

Excluding the step followe' hy an asterisk, $\mathrm{CHl} 2=\sum_{l=1}^{N}\left(p h(i)-y(i)-\delta_{11}\right)^{2}$. The "asterist step" essentially adds $2 \pi$ times an integer to the difference between the actual phase and the phase fit, so that $D \leq \pi$. Two signals cannot be more than $180^{\circ}$ out of phase, and adding any integer times $2 \pi$ to a phase does not change the signal it represents. 
$\delta_{0}$ could be solved for by applying, a "grid search" followed by an iterative minimization routine using the $\mathrm{CH} 2$ function with $\delta_{0}$ restricted to lic within any $2 \pi$ interval. In order to determine $m$ and $n$ this minimization algorithm is placed within two nested DO loops, which test each $m, n$ combination and minimize CHI2 $=\mathrm{CH} 12\left(m, n, \delta_{0}\right)$ with $\lambda^{\prime}$ given. The resulting minimur CHI2 value defines CHI2( $\left.\lambda^{\prime}\right)$, and (if desired) the optimum $\lambda^{\prime}$ value may be determined by applying a "search and iterate" routine to this CHI2( $\lambda$ ') function. Analysis of Mirnov data may typically involve running this four parameter search algorithm very often. Since the algorithm to determine $\delta_{0}$ is nested within loops to determine $m, n$ and $\lambda^{\prime}$, it would be beneficial to speed up the mitethod of solving for $\delta_{0}$, given $m, n, \lambda^{\prime}$.

An analytical method was developed to find the value of $\delta_{t,}$ which will minimize $\mathrm{CHI}\left(\delta_{0}\right)$ with $m, n, \lambda^{\prime}$ given. It can best be described by defining an equivalent norm, CHINEW $\left(\varepsilon_{i i}\right)$. Let

$$
\tau(i)=p h(i)-\left\{m\left|\theta(i)+\lambda^{\prime} \sin \theta(i)\right|+\phi(i)\right\} .
$$

Let $\{t(i)\}_{i=1}^{N}$ be the sequence $\{r(i)\}_{i=1}^{N}$ with each element mapped into the interval $i-\pi, \pi !$ by adding $2 \pi$ times an integer. Let $\left\{W_{0}(i)\right\}_{i=1}^{N}$ be the $\{t(i)\}_{i=1}^{N}$ sequence rearranged in ascending order. Now $\mathrm{CH} 2\left(\delta_{G}\right)=\mathrm{CHJ} 2\left(y, p h_{n} n, \delta_{i}\right)$ may be defined equivalently in terms of the $\left\{W_{n}(i)\right\}_{S_{2}=1}^{N}$ sequence.

$$
\begin{aligned}
& \text { FUNCTION CHINEW }\left(W_{0}, N, \delta_{0}\right) \\
& \text { CHINEW }=0 . \\
& \text { DO } 10 \mathrm{i}=1, N \\
& \mathrm{D}=\mathrm{ABS}\left(W_{0}(i)-\delta_{0}\right) \\
& \mathrm{D}=\mathrm{AMOD}(\mathrm{D}+\pi, 2 \pi)-\pi \\
& 10 \text { CHINEW }=\mathrm{CHINE} W+\mathrm{D} * \mathrm{D} \\
& \text { RETLRN }
\end{aligned}
$$

Sote CHINEW $\left(W_{i 1}, N, \delta_{10}\right)=\operatorname{CHI} 2\left(\delta_{11}\right)$. Note if $\delta_{01} \in\left[W_{0}(N)-\pi, W_{0}(1)+\pi\right]$, then the "asterisk step" has no effect, CHINEW $=\sum_{i=1}^{N}\left(W_{0}(i)-\delta_{0}\right)^{2}$, and by slandard analysis, $\delta_{0}=\frac{1}{N} \sum_{i=1}^{N} w_{0}(i)=\bar{W}_{0}$ is a critical point of CHINEW.

Define another sequence, $\left\{W_{1}(i)\right\}_{i=1}^{N}=\left\{w_{0}(2), W_{0}(3), \ldots, w_{0}(N), W_{0}(1)+2 \pi\right\}$. The elements of $\left\{W_{1}(i)\right\}_{i=1}^{N}$ are in ascending order. Note CHINEW( $\left.W_{1}, N, \delta_{0}\right)=\operatorname{CHI}\left(\delta_{0}\right)$. Note if $\delta_{0} \in\left[W_{0}(1)+\pi, W_{0}(2)+\pi\right]$, then the "asterisk step" has no effec, CHINEW $=$ $\sum_{i=1}^{N}\left(W_{1}(i)-\delta_{i j}\right)^{2}$, and by standard analysis $\delta_{i 1}=\frac{1}{N} \sum_{i=1}^{N} W_{1}(i)=\overline{W_{0}}-\frac{2 \pi}{N}$ is a critical point.

Define the 1:ext sequence, $\left\{W_{2}(i)\right\}_{i=1}^{N}=\left\{W_{0}(3), \ldots, W_{0}(N), W_{v}(1)+2 \pi, W_{0}(2)+2 \pi\right\}$. The elements of $\left\{W_{2}(i)\right\}_{i=1}^{N}$ are in ascending order. Note CHINEW $\left(W_{2}, N, \delta_{0}\right)=\operatorname{CHI} 2\left(\delta_{0}\right)$. 
Note if $\delta_{0} \in\left\{W_{0}(2) \div \pi, W_{0}(3)+\pi\right\}$, thest the "asterisk step" has no effect, CHINEW= $\sum_{i=1}^{N}\left(W_{2}(i)-\delta_{0}\right)^{2}$, and by standard analysis $\delta_{i j}-\frac{1}{N} \sum_{i=1}^{N} W_{2}(i)=\overline{W_{0}}+\frac{4 \pi}{N}$ is a critical point.

Continuing to add $2 \pi$ to the first and least element of the previous ascending $W_{k}$ sequence and transferring the result to the last (and greatest. ' element of the $H_{k+1}$ sequence generates $\mathrm{N}$ critical points over a $2 \pi$ interval (or domain) for $\delta_{11}$. The critical points are $\left\{c_{k}\right\}_{k=0}^{N-1}=\left\{\overline{W_{0}}+\frac{2 \pi k}{N}\right\}_{k=0}^{N-1}$. It has been rigorously proven that the optimal $\delta_{0}$ is in this set of critical points and if $\delta_{0}=c_{k}$ is cptimal, then $\mathrm{CHI} 2\left(y, p h, N, c_{k}\right)=\sum_{i=1}^{N}\left(W_{k}(i)-c_{k}\right)^{2}$.

Generating the sequence $\left\{H_{k}\right\}_{k=0}^{N-1}=\left\{\sum_{i=1}^{N}\left(W_{k}(i)-c_{k}\right)^{2}\right\}_{k=0}^{N-1}$ may be simplified by substituting for $c_{k}$ and the $W_{k}$ 's in terms of the $W_{0}$ 's. A recurrence relation useful for determining the smallest eftment of the sequence, $\left\{H_{k}\right\}_{k=0}^{N-1}$ : may then be derived. Substituting $c_{k}=\overline{W_{0}} \div \frac{2 \pi k}{N}$ and $\sum_{i=1}^{N} W_{0}(i)=N \overline{W_{i}}$ into

$$
\begin{aligned}
& \left.H_{k}=\sum_{i=1}^{k}\left[W_{0}(i)+2 \pi-c_{k}\right]^{2}+\sum_{i=k+1}^{N} \mid w_{11}(i)-c_{k}\right]^{2} \\
& \text { and letting } S_{k}=\frac{1}{4 \pi}\left[H_{k}-\sum_{i=1}^{N} W_{0}^{2}(i)-N\left(\bar{l} w_{1}\right)^{2}{ }_{i} .\right.
\end{aligned}
$$

one finds that $S_{0}=0$ and for $k>0$,

$$
S_{k}=W_{0}(k)-\overline{W_{0}}+\frac{2 \pi}{N}\left(\frac{N-1}{2}-k\right)+S_{k-1}
$$

Finding $j$ such that

$$
S_{j}=\min _{n \leq k \leq y_{-1}} S_{k}
$$

determines the optimal $\delta_{0}, i . e_{1}, \delta_{0}=c_{j}$. The corle to implement this algorithm for determining $m, n, \delta_{0}$ for a given $\lambda^{\prime}$ is given in the appendix.

The described algorithm was used to define CHI2 $\left(\lambda^{\prime}\right)$ whicn minimizes CHI2 with respect to $m, n, \delta_{i}$. The described algorithm was also used 10 minimize $C H J 2$ with resper: to all four parameters, $m, n, \delta_{t}, \lambda^{\prime}$. CHI2 $\left(\lambda^{\prime}\right)$ was evalualed at 32 equally spaced points over the region of interest, $\left(-1 \leq \lambda^{\prime} \leq 1\right)$, to constrict the nejghborhood containing the absolute minima. This prevented the subsequant iterative mininization routine from converging to any relative minima other than the absolute minima.

Another method of minimizing CHI2 with respect to all four parameters was devised. Rather than applying a search and standard iterative minimization routine using CHI?( $\left.\lambda^{\prime}\right)$ 
to determine the optimal $m, n, \delta_{0}, \lambda^{\prime}$, a much more sophisticated code was developed to find both $\lambda^{\prime}$ and $\delta_{0}$ analytically. For large $N$ and a fixed range of $m, n$ numbers the more sophisticated algorithm's run time scaled as $N^{3}$ and the described iterative approach scaled as $N^{2}$. Unfortunately, for TFTR data ( $N=19$ Mirnov coils) the sophisticated analytical algorithm rán only slightly faster than the iterative algorithm when allowing a 0.00001 error in $\lambda^{\prime}$. For the algorithm which obtained both $\lambda^{\prime}$ and $\delta_{0}$ analytically, the added complexity and 200 lines of FORTRAN code required did not seem to be justified.

A second method for finding both $\lambda^{\prime}$ and $\delta_{0}$ analyically exists. This optional method was not used to analyze the Mirnov data. Unlike the method described in the preceding paragraph, this method is not certain to provide the corsect answer when one or mose "bad" data points are approximately $18 \mathrm{C}^{\circ}$ away from the optimal phase fit. This method involves iteratively minimizing $\mathrm{CHI} 2\left(\lambda^{\prime}\right)$ until one has determined the $m, n$ numbers and has a goo'd approximaliun of what $\lambda^{\prime}$ and $\delta_{0}$ are. Let the parameters obtained at the end of this iterative minimization procedure be $\left(m_{g}, n_{g}, \delta_{g}, \lambda_{g}^{\prime}\right)$. Let $s\left(\delta_{i}, \lambda^{\prime}, i\right)=\left|m_{g}\left(\theta(i)+\lambda^{\prime} \sin \theta(i)\right\}+n_{g} \phi(i)+\delta_{0}-\overline{p h}(i)\right|^{2}$ wherc $p h(i)$ is the value of $p h(i)$ summed with the appropriate multiple of $2 \pi$ to make $s\left(\delta_{0}=\delta_{g}, \lambda^{\prime}=\lambda_{g}^{\prime}, i\right) \leq \pi^{2}$. Let ting

$$
j=f\left(\delta_{0}, \lambda^{\prime}\right)=\sum_{i=1}^{N} s\left(\delta_{i}, \lambda^{\prime}, i\right)
$$

the optimal values of $\lambda^{\prime}$ and $\delta_{0}$ are then found by setting

$$
\frac{\partial f}{\partial \delta_{0}}=\frac{\partial f}{\partial \lambda^{\prime}}=0
$$

The resulting parameters are $m=m_{g}, n=n_{g}$ and with $x(i)=-\dot{p h}(i)+m \theta(i)+n \dot{\phi}(i)$,

$$
A=\sum_{i=i}^{N} x(i), B=\sum_{i=1}^{N} x^{2}(i), C=\sum_{i=1}^{N} \sin \theta(i), D=\sum_{i=1}^{N} \sin ^{2} \theta(i), E=\sum_{i=1}^{N} x(i) \sin \theta(i),
$$

one obtains

$$
\left(\begin{array}{c}
\delta_{0} \\
m \lambda^{\prime}
\end{array}\right)=\frac{1}{D N-C^{2}}\left(\begin{array}{c}
C E-A D \\
A C-E N
\end{array}\right)
$$

and for these parameters, $\mathrm{CHI} 2=f=B+E m \lambda^{\prime}+A \delta_{0}$. An advisable precaution would be to use this method to terminate the $\mathrm{CHI} 2\left(\lambda^{\prime}\right)$ minimization iteration only if this method yields the same answer when $\left(m_{g}, n_{g}, \delta_{g}, \lambda_{g}^{\prime}\right)$ are set to the values corresponding to the minimum and maximum values within which $\lambda^{\prime}$ has been restricted to lie. 


\section{CONCLUSION}

The methods presented for constructing phase fits and polar plots significantly improved the accuracy of mode number detemination frorn Mirnov coil data. It was found that making a proper fit to the phase data required a correction parametes, $\lambda^{\prime}$, to accommodate the effects of the toroidicity and the off-center position of the mode. Improved resolution of mnde geometry was made possible through better data analysis techniques so that modes with $m$ numbers as high as $m=9$ can be clearly seen on the various plots presented.

Interesting physical phenomena occurring during the start-up of shot \#9689 were observed. As the mode numbers evolved from higher to lower $m$ numbers, seven different modes with $n=1$ and $m$ numbers from $m=9 \pm 0 m=3$ were detected. A correlation between $m / n$ and $q_{s}(a)$ was observed. Minor disruptions were preceded by growing modes. Simultaneously exjsting modes were found with $m=4, n=1$ and $m=3, n=1$. The modes detected on shot $\# 9689$ and other shots consistently rotated in the electron diamagnetic direction. More analysis of Mirnov coil data would be useful to see if the physical phenomena seen on shot $\# 9689$ are typical.

Mirnov data from the different modes were used to construct polar plots which rlearly displayed increased spatial variation on the inner side of the tokamak. Placing relatively more Mirnov ccils on the inner side of the machine should be considered for future macbine designs in order to enhance mode resolution and the detection of high $m$ numbers. The effect of toroidicity typically dominates the effect due to the mode's off-center position for TFTR data. Theoretical calculations from Merczhkin ${ }^{2}$ on this toroidal effect were tested and found to be consistent with the data.

Anyone who wishes to obtain $m, n$ numbers from phase correlation analysis could benefit from the algorithm which was developed for that puspose. The difficulties in estimating the value of $\lambda^{\prime}$ were circumvented by using the computes to find $\lambda^{\prime}$ automatically. The improved efficiency of the algorithm reduces ruл time greatly and significantly enhances the ease of between-shot analysis requiring $m, n$ number calculation.

\section{ACKNOWLEDGMENTS}

The author wishes to thank Gregory Hammett for many helpful discussions. This work was supported by U.S. Department of Energy Contract No. DE-AC02-76-CHO-3073. 


\section{APPENDIX}

$C$ - Heuristic code to determine $m, n, \delta_{0}$ for a given $\lambda^{\prime}$

$\mathrm{C}$ - and return the resulting $\mathrm{CHI} 2\left(\lambda^{\prime}\right)$ value

FUNCTION CHI2( $\left.p h, \theta, \phi, \lambda^{\prime}, \mathrm{MLOW}, \mathrm{MHI}, \mathrm{NLOW}, \mathrm{NHI}, \quad m, n, \delta_{0}\right)$

$\mathrm{P}=\mathrm{FLOAT}(\mathrm{N}+1) / 2$.

$\mathrm{Q}=2 \pi / \mathrm{FLOAT}(\mathrm{N})$

DO $5 i=1, N$

$5 \quad \operatorname{SI}(i)=\sin (\theta(i))$

$\mathrm{CHI} 2=1000$.

DO $30 \mathrm{M} 1=\mathrm{MLOW}, \mathrm{MHI}$

DO $30 \mathrm{~N} 1=\mathrm{NLOW}, \mathrm{NHI}$

WSUM $=0$.

WWSUM = 0.

DO $10 i=1, N$

$\left.W(i)=p h(i)-F L O A T(M 1) *\left(\theta(i)+\lambda^{\prime} * \operatorname{SI}(i)\right)-F I O A T(N 1) * \phi(i)\right) \quad$ ! (see eqn.1)

$\mathbf{Z}=\pi$

IF(W(i).LT.0.)Z $Z=-\pi$

$W(i)=\operatorname{AMOD}(W(i)+Z, 2 \pi)-Z$

WSUM $=W S U M+W(i)$

$10 \quad W W S U M=W W S L M+W(i) * W(i)$

WAVG $=$ WSUM/FLOAT $(\mathrm{N})$

$C$--_-Subroutine ORDER arranges the $W$ array in ascending order

CALL ORDER(W,Ni)

$S=0$.

SBEST $=S$

$\mathrm{j}=0$

DO $20 k=1, N-1$

$\mathrm{SNEXT}=W(k)-W A V G \div Q *(\mathrm{R}-\mathrm{FLOAT}(k))+\mathrm{S}$ ! (see eqn 3 )

IF(SNEXT.GE.SBEST) GOTO 20

SBEST $=$ SNEXT

$\mathrm{j}=k$

$20 \quad S=S X E X T$

$\mathrm{CHI}=4 \pi *$ SBEST + WWSUM - WSUM $*$ WAVG I (see eqn.2)

IF(CHI.GE.CHI2) GOTO 30

$\mathrm{CH} 12=\mathrm{CHI}$

$\delta_{1}=$ WAVG $-Q *$ FLOAT $(\mathrm{j})$

$m=M 1$

$n .=\mathrm{N} 1$

30. CONTINUE

RETURY

If desired, one could further optimize the program by setting up arrays PHN1 $(i, N 1-N L O W+1)=\phi(i) * F L O A T(N 1), \operatorname{THM} 1(i, M 1-M L O W+1)=\theta(i) *$ LOAT $(M 1)$, 
and SINM1 $(i, M 1-M L O W+1)=\sin (\theta(i)) *$ FLOAT(M1). Passing PHNi, THM1, SINM1, $R$, and $Q$ to FUNCTION CHI2 through a common block would then enabie the $W$ array to be initialized with less multiplication and reduce needless redundancy in the computation, (especially if FUNCTION CHI2 is called more than once). Note minor modifications of this code would be required for a FORTRAN compiler, i.e., $\pi, \theta, \phi, \delta_{0}$, and $\lambda^{\prime}$ need new names and EXTERNAL, DIMENSION, and COMMON statements should be included. The MSL Library" provides a routjue called VRSTA which arrange arrays in ascending order.

\section{REFERENCES}

1. V. D. Shafranot, Reviews of Plasma Physics [2] The Consultants Bureau, New York, (1969) 103 .

2. V. G. Merezhkin, Sov. J. Plasma Phys. 4 \{2) (1978) 152.

3. G. Hammetr and K. McGuire, Princeton Plasma Physics Lab. Rep. PPPL-1854, 15 (1982).

4. R. S. Granetz, I. H. Hutchinson, D. O. Overskei, Nuclear Fusion 19 (3) (1979) 1587.

5. R. B. White, "Resistive Instabilities and Field Line Reconnection." In Vol. 1 of Basic Plasma Physics, edited by A. A. Galeev and R. N. Sudan, North Holland Publishing Co., New York, (1983) p.632-633.

6. L. C. J. M. DeKock, B. J. H. Meddens, L. T, M. Ornstein, D. C. Schram. R. J. J. Van Heijningen, Massachusetts Institute of Technology Rep. PRR-7414 (1974).

i. G. Fussman. B. J. Green, H.P. Zehrfeld, Plasma Physics and Controlled Nuclear Fusion Research (Proc. Int. Conf. Brussels, 1980).

8. Phillip R. Bevington, Data Reduction andi Error Analysis for the Plysical Sciences, McGraw-Hill, New York, (1969).

9. IMSL Inc., The IMSL Library Edition 9 Reference Montal i2: International Mathematical and Statistical Libraries, Inc., Houston, (1982). 
Table 1.

\begin{tabular}{|c|c|c|c|c|c|}
\hline time range $(\mathrm{ms})$ & freq $(\mathrm{kHz})$ & $m, n$ & $q_{s}(a)$ & $\delta B_{0} / B_{0} \%$ & $\chi_{\lambda^{\prime}}^{2}$ \\
\hline $85-90$ & 1.4 & 9,1 & 9.3 & 0.057 & 0.193 \\
\hline $120-130$ & 1.1 & 8,1 & 7.8 & 0.055 & 0.065 \\
\hline $190-200$ & 0.8 & 7,1 & 6.8 & 0.152 & 0.059 \\
\hline $285-290$ & 1.5 & 5,1 & 6.3 & 0.021 & 0.062 \\
\hline $382-387$ & 1.2 & 6,1 & 6.0 & 0.052 & 0.048 \\
\hline $530-535$ & 1.2 & 3,1 & 5.5 & 0.062 & 0.033 \\
\hline $540-550$ & 0.8 & 4,1 & 5.4 & 0.126 & 0.016 \\
\hline
\end{tabular}


Table 2.

\begin{tabular}{|c|c|c|c|c|c|c|c|c|}
\hline timc range (ms) & freq $(\mathrm{kHz})$ & $m, n$ & $q_{s}(a)$ & $\delta B_{0}{ }^{\prime} B_{0} \%$ & $\chi_{\lambda^{\prime}}^{2}$ & $\chi_{\lambda_{\text {mode }}^{\prime}}^{2}$ & $\lambda_{\text {mode }}^{\prime}$ & $\lambda_{\text {Marez }}^{\prime}$ \\
\hline $107 \cdots 111$ & 2.0 & 8,1 & 8.3 & 0.037 & 0.111 & 0.100 & 0.368 & 0.443 \\
120130 & 1.1 & 8,1 & 7.8 & 0.055 & 0.065 & 0.045 & 0.432 & 0.445 \\
135138 & 1.7 & 8,1 & 7.5 & 0.057 & 0.132 & 0.113 & 0.438 & 0.442 \\
\hline $145-150$ & 1.2 & 7,1 & 7.2 & 0.085 & 0.127 & 0.135 & 0.323 & 0.441 \\
$190-200$ & 0.8 & 7,1 & 6.8 & 0.152 & 0.059 & 0.039 & 0.460 & 0.458 \\
$210-220$ & 0.7 & 7,1 & 6.7 & 0.149 & 0.066 & 0.051 & 0.484 & 0.467 \\
\hline $330-335$ & 1.4 & 6,1 & 6.2 & 0.075 & 0.054 & 0.045 & 0.436 & 0.469 \\
$510-350$ & 1.1 & 6,1 & 6.1 & 0.106 & 0.052 & 0.043 & 0.450 & 0.483 \\
382387 & 1.2 & 6,1 & 6.0 & 0.052 & 0.048 & 0.038 & 0.472 & 0.491 \\
\hline
\end{tabular}




\section{FIGUKE CAPTIONS}

Fig. 1 Locations of Mirnov coils in TFTR.

Fig. 2 Examining a Mirnov signal from shot \#9689 reveals a region of interest (a), which is expanded and compared with the signals from the poloidal array of the first 16 Mirsov coils (b).

Fig. 3 This figure shows how the data shown in Fig. 2 are analyzed. Each signal is Fourier analyzed from $340-350 \mathrm{~ms}$ and each signal's Fourier spectrum of amplitudes is averaged together (a). The phases from rach coil at $1.1 \mathrm{kHz}$ are shown in (b). The data points from coils 17-19 are usually denoted by a distinctiv symbol in this report. The term $\pi[\phi(1)-\phi(i)]$ is added to the phases from coils 17-19 in order to compare their phases with the polridal array which is at a different toroidal angle. This term comes from the 0 dependence in the $\xi=m\left(\theta+\lambda^{\prime} \sin \theta\right)+n \phi+\delta_{0}$ model. Thus an $n=1$ number was assumed a priori in (b) in order to plot the data from coils 17-19. The phase data in (b) are correlated by an optimal cylindrical fit (c). An optimal toroidal fit (d), in which $\lambda^{\prime}$ is chosen to optimize the fit, is also shown.

Fig. 1 The dashed line inficates the optimal cylindrical fit to a typical $m=4$ mode's phase data. T,e cylindrical fic "wraps around" from bottom to top 3 times, indicating $m=3$. The optimal toroidal fit corrertly indicates $m=4$.

Fig. 5 Four different ways of analyzing the same set of phase data are illustrated. The cylindrical fit (a) indicates $m=4$. Interpolating $\{\theta(i), r(i)=a(i) \cos \{p h(i)\}+C\}_{i=1}^{N}$ with a cubic spline (b) indicates $m=5$. Th" optimal toroidal fit (c) correctly indicates $m=\epsilon$. Careful analysis of the $n=6$ corrected polar plot (d) reveals that a branch of the mode was missed in (b). The location of this branch is indicated by an arrow in (b).

Fig. 6 The phase data in (a) From is $m=6$ mode are adjusted by adding appropriate multiples of $360^{3}$ in order to illustrate the optimal cylindrical and toroidal fits in a continuous manner. The optimal toroidal fit. is adjusted to interpolate the phase data by adding the cubic spline shown in (b). A cubic spline of the amplitude data taken from each coil at the same frequency is shown in (c). These cubic splines have matching $0^{\text {th }}$, $1^{\text {at }}$, and $2^{\text {nd }}$ derivatives at $\theta=0^{\circ}$ and $\theta=360^{\circ}$ and were calculated by IMSL $L^{\circ}$ routine ICSPLN. The corrected polar plot which (b) and (c) were used to produce is shown in (d). The same polar plot data against $\theta=\theta+\lambda^{\prime} \sin \theta$ (instead of $\theta$ ) are shown in (e).

Fig. 7 Parameters of shot \#9689 during slart-up including $\Lambda=\beta_{n}-l_{1} / 2, R_{p}, J_{p}$, and $q_{i}(a)$.

Fig. 8 Line-averaged density of shot $\ddot{\#} 9689$ during start-up. 
Fig. 9 A summary of the mode evolution during start-up on shot \#9689 is shown with the Mirnov signal from coil \#9.

Fig. 10-11 Phase data from 7 of the modes detected during start-up are illustrated. Additional data foi these 7 modes are shown in Table 1. The 7 modes in Figs. 10-11, Figs. 12-13, Fig. 14 and Table 1 were each taken from the same time range and frequency.

Fig. 12-13 Polar plots for the 7 detected modes. Although $m=9$ is above the Nyquist limit for 16 evenly spaced Mirnov coils, the 3 other coils remove the "degeneracy" between the $m=9$ and $m=-7$ modes. For the phase data of the given $m=9$ mode, $\chi_{\lambda^{\prime}}^{2}(m=9, n=1)=0.193<\chi_{\lambda^{\prime}}^{2}(m=-7, n=1)=0.241$.

Fig. 14 The phases from the 7 modes have been negated and shifted by appropriate multiples of $360^{\circ}$ in order to show the data and their optimal toroidal phase fits in a cont inuous mannet.

Fig. 15 The effects of disruptive plasma behavior in the Mirnov coil and loop voltage signals during shot.

Fig. 16 Parameters of the plasma during the disruptive activity shown in Fig. 15.

Fig. 17 Simulaneous $m=3$ and $m=4$ modes were detected at $1.0 \mathrm{kHz}$ and 0.8 hHz, respectively.

Fig. 18 Polar plot for the sinultaneous $m=3$ and $m=4$ modes.

Fig. 19 The lower 3 diagrami illustrate an $m=3$ mode rotating wi ${ }^{\prime} h_{1}$ time in the electron diamagnetic drift direction during shot \#9689. The upper 3 diagrams show an $m=3$ mode's motion during another shot (\#9031) with the electron diamagnetic drift direction reversed.

Fig. 20 This picture illustrates the variables used to aftalyze the effect of the mode's off-center position on the phase data. The transformation from coordinates taken with respect to the vacuum vessel to coordinates relative to the center of the modr is derived from $\theta^{\text {mode }}=\theta+\alpha$ and $\sin \alpha / d=\sin \theta / \sqrt{b^{2}+d^{2}-2 b d \cos \theta}$

Fig. 21 The curvet solid line indicates the polar plot of the actual data. The asymmetrically increased spatial variation in the phase data on the inner side of the tokamak is caused by toroidal sffects and the off-center position of the mode relative to the vicuum vessel center. The dashed line shows the same radjal data with the poloidal coordinates taken relative to the center of the mode, thus attempting to remove the effect due to the off-centcr position. Since the dashed outline of the mode shows that the noncylindrical asymmetry of the mode is only slightly reduced, it illustrates that the toroidal effect dominates the effect due to the mode's off-center position. 


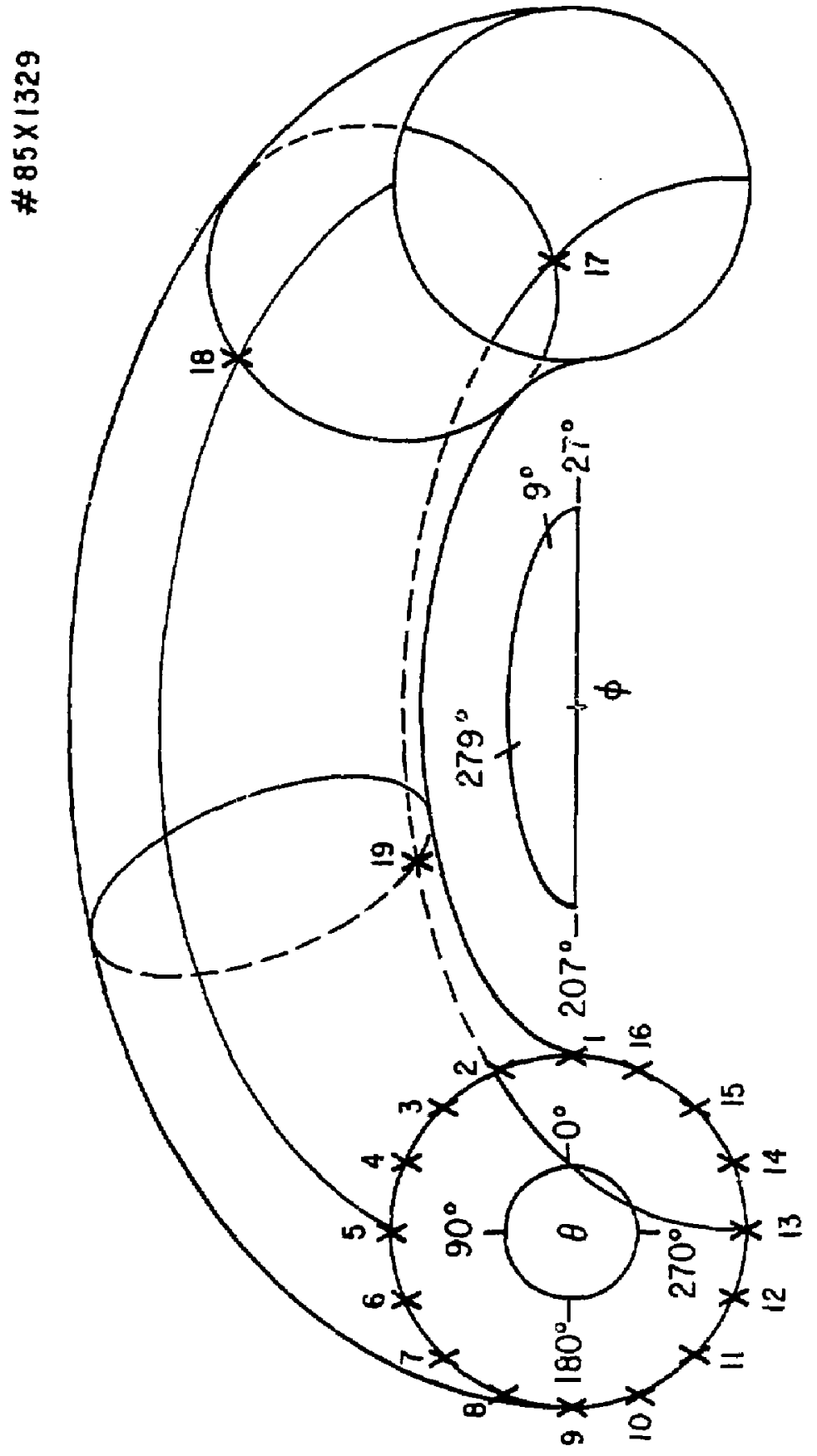



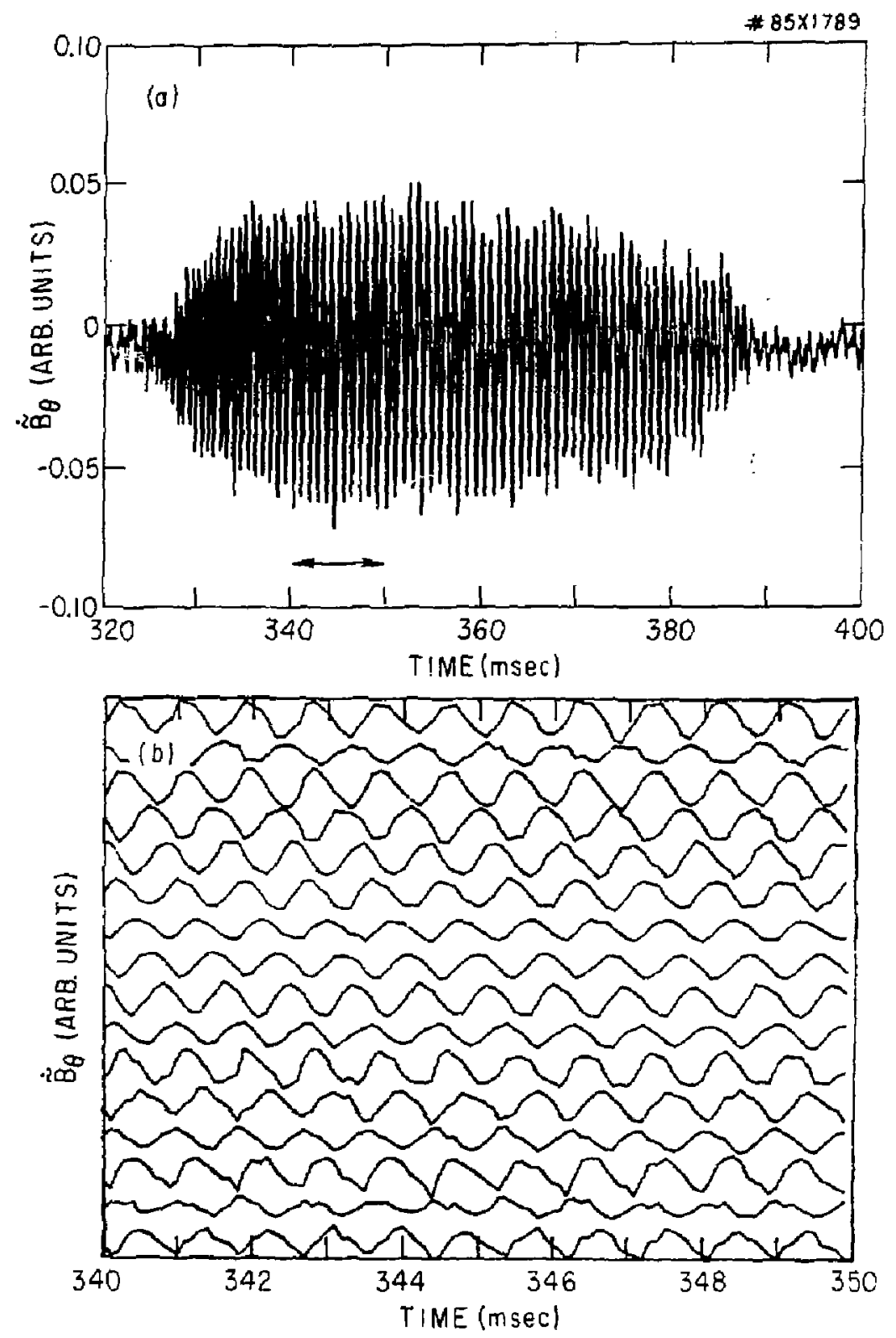

Fig. 2 


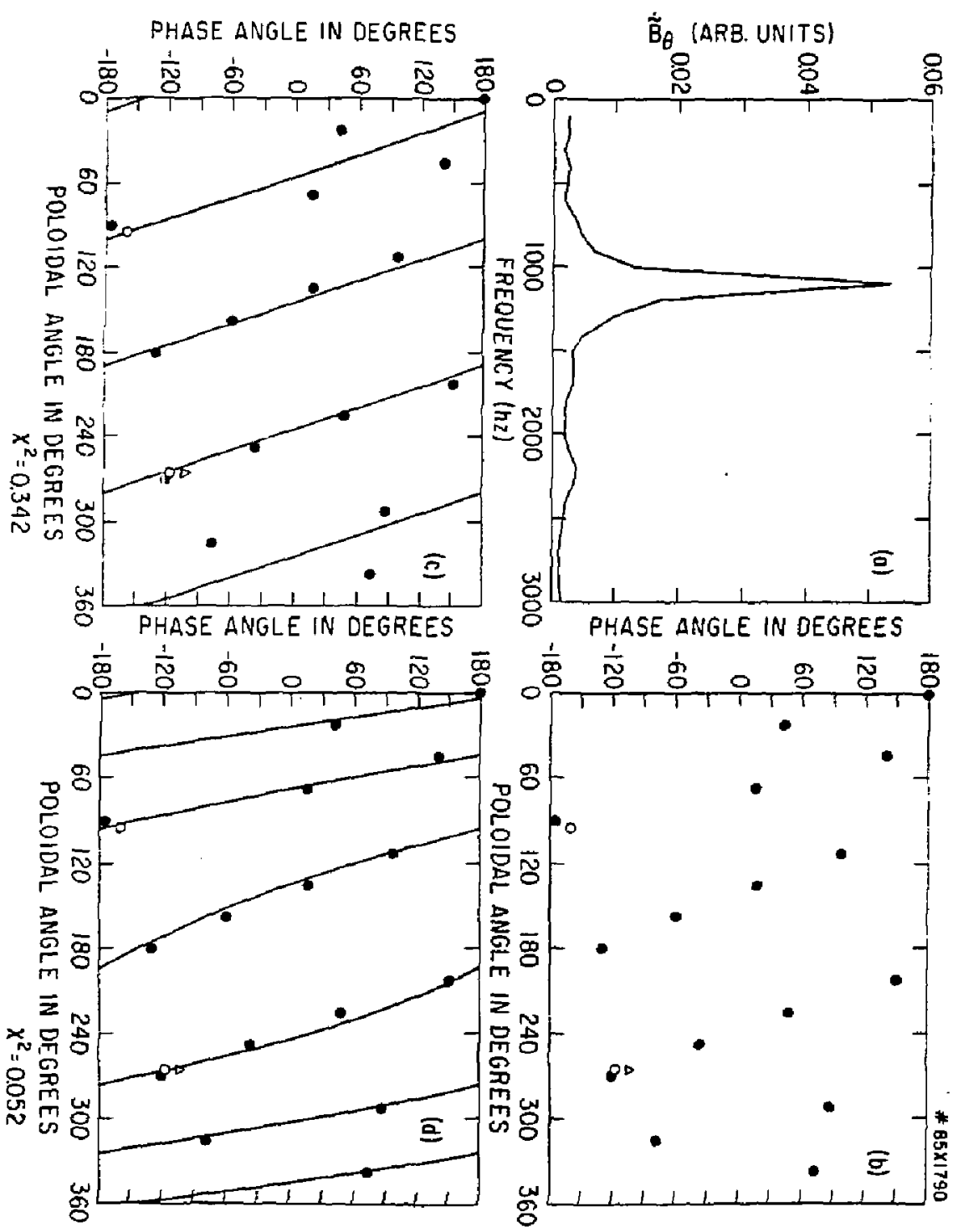




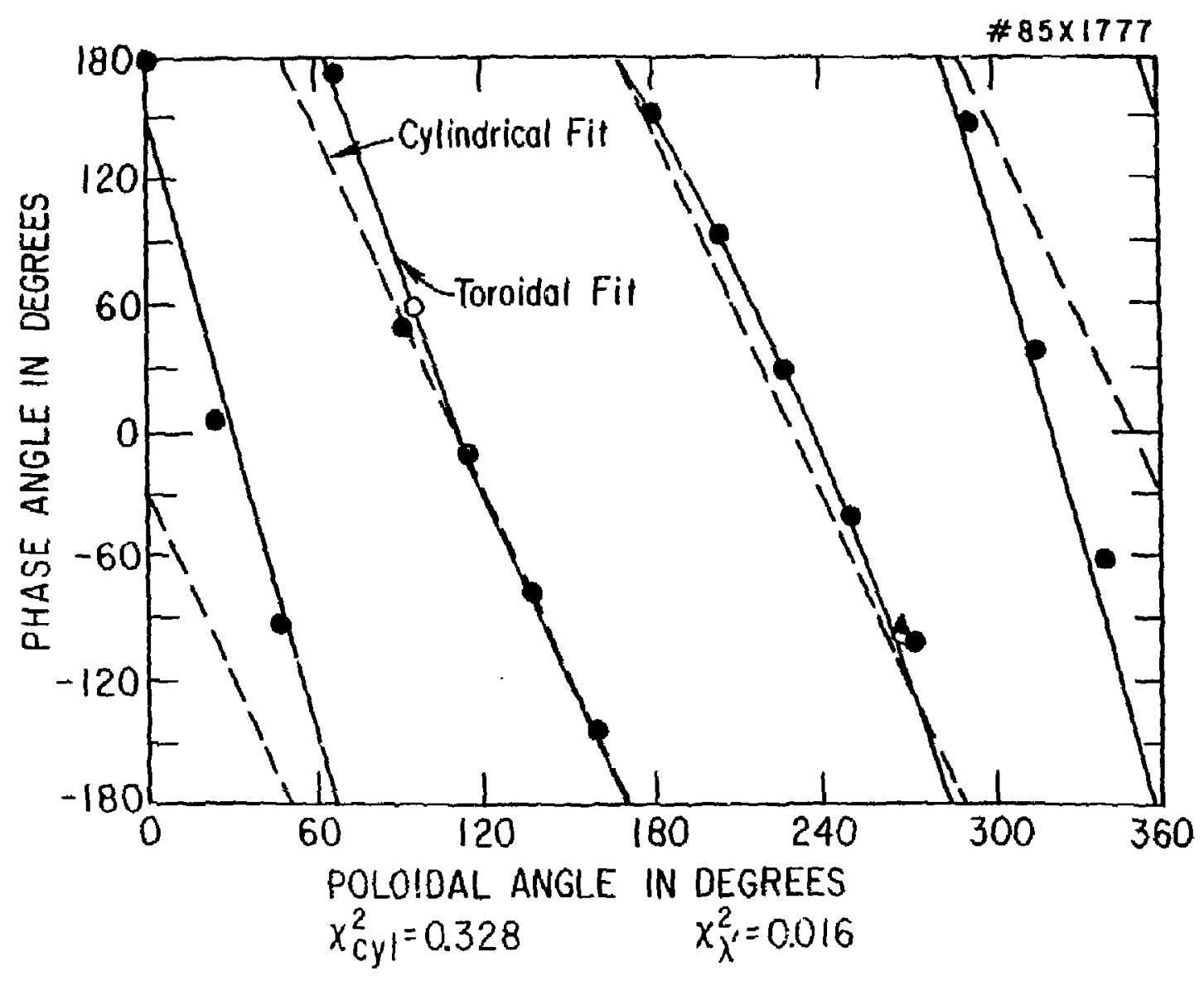



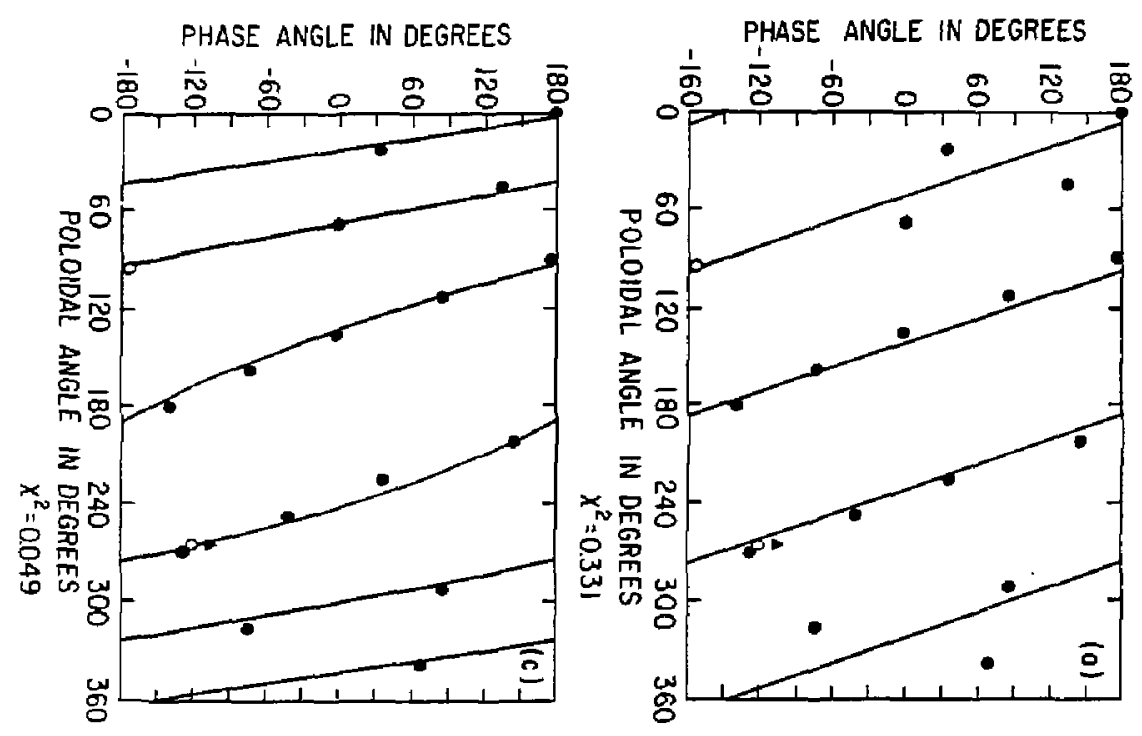

$=$
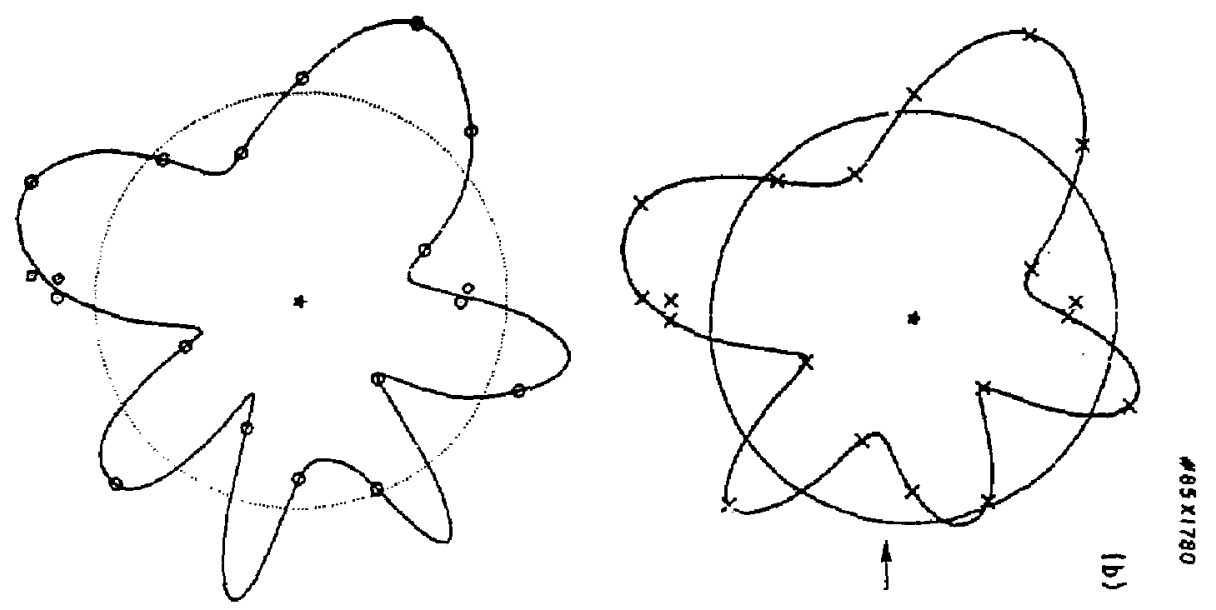

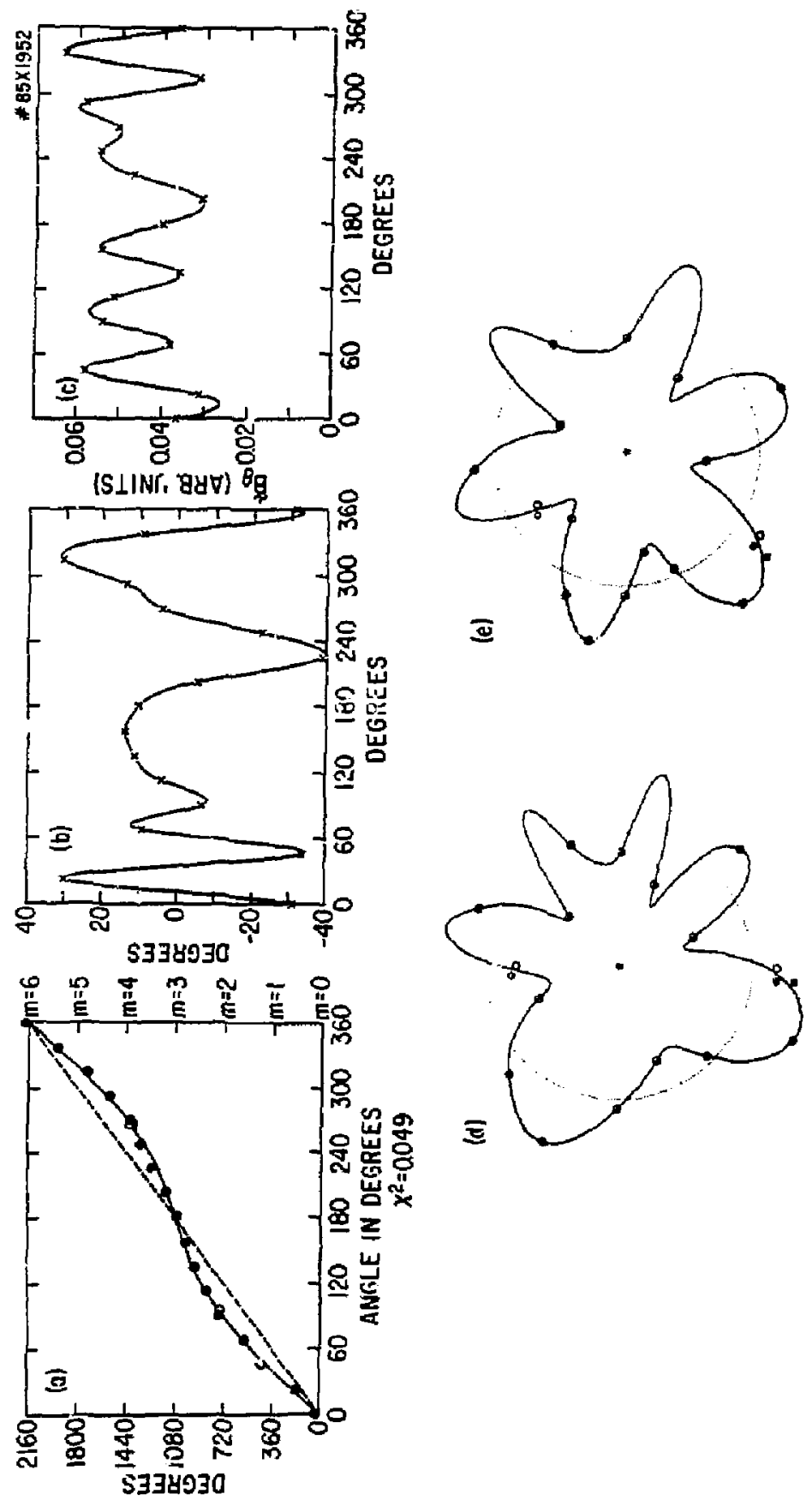

$\underset{\square}{\dot{\theta}}$ 

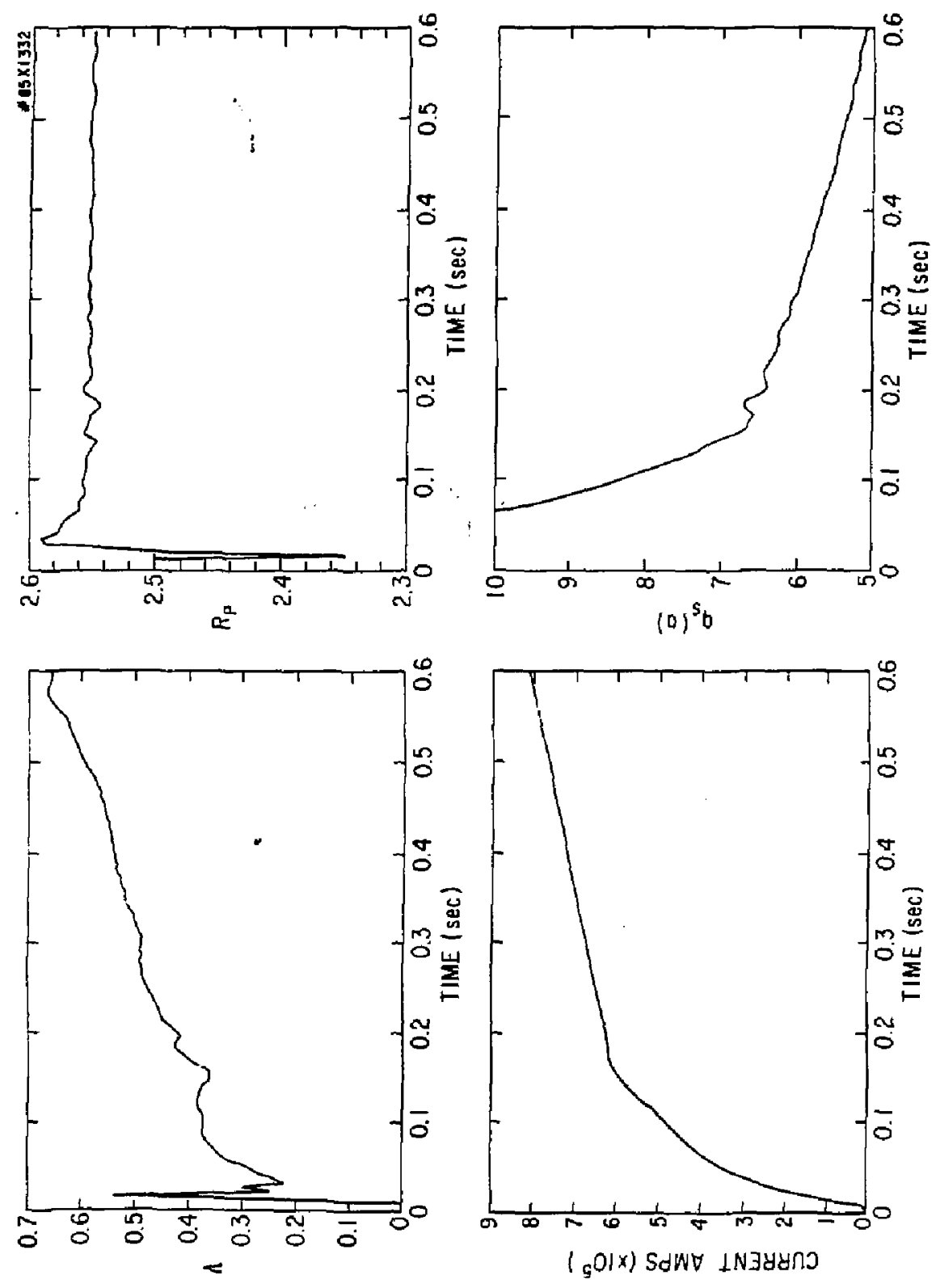


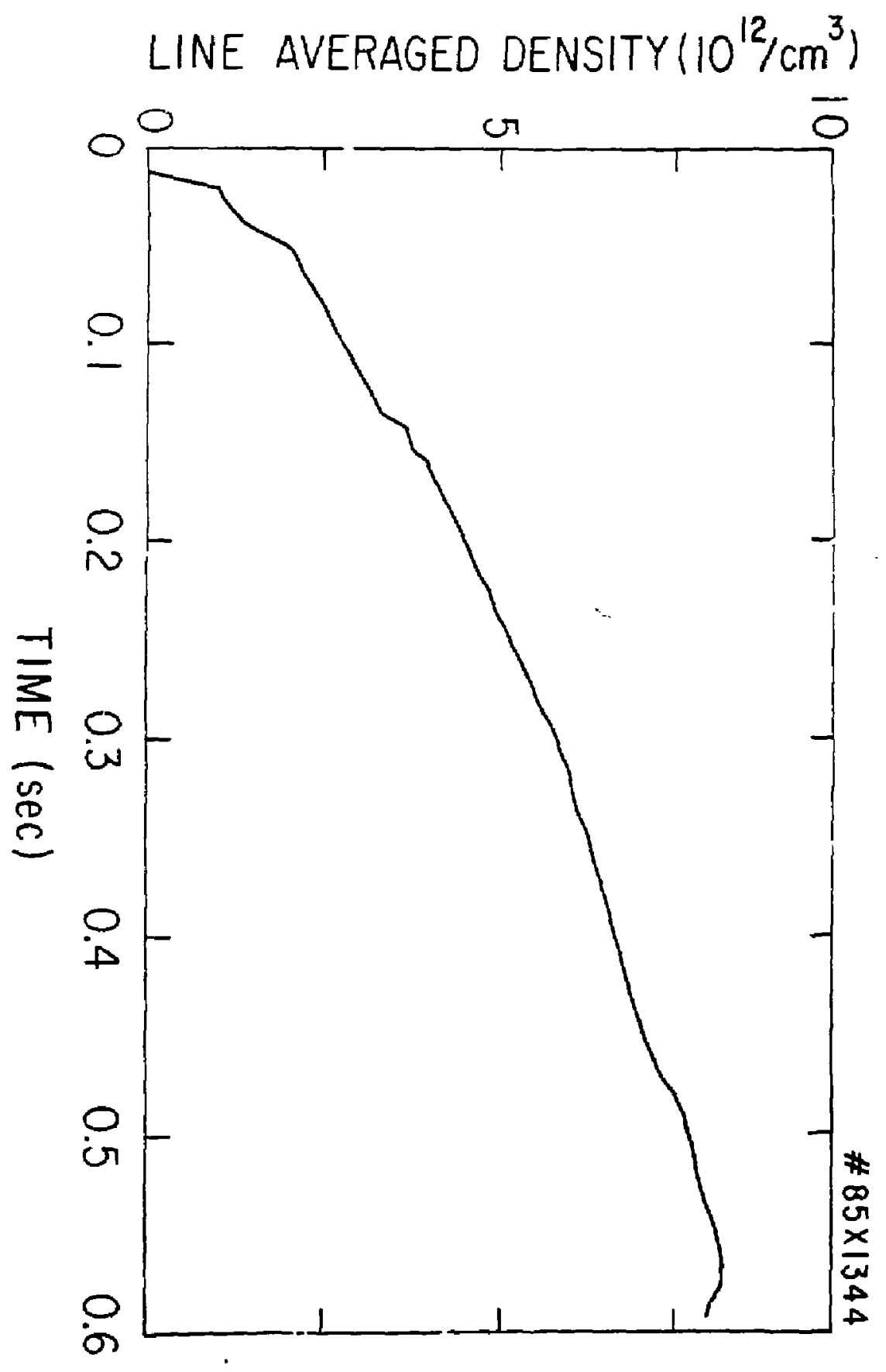




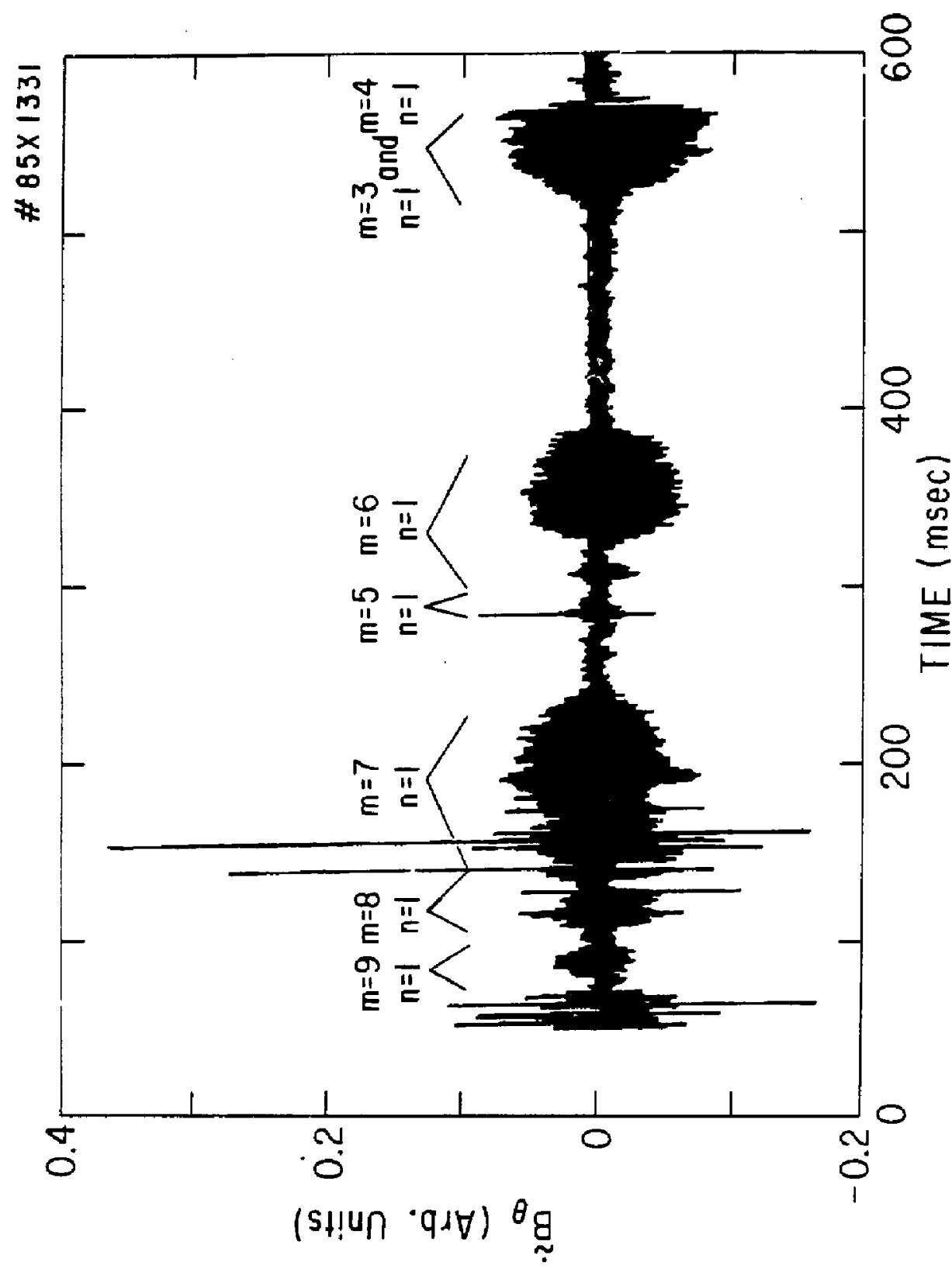



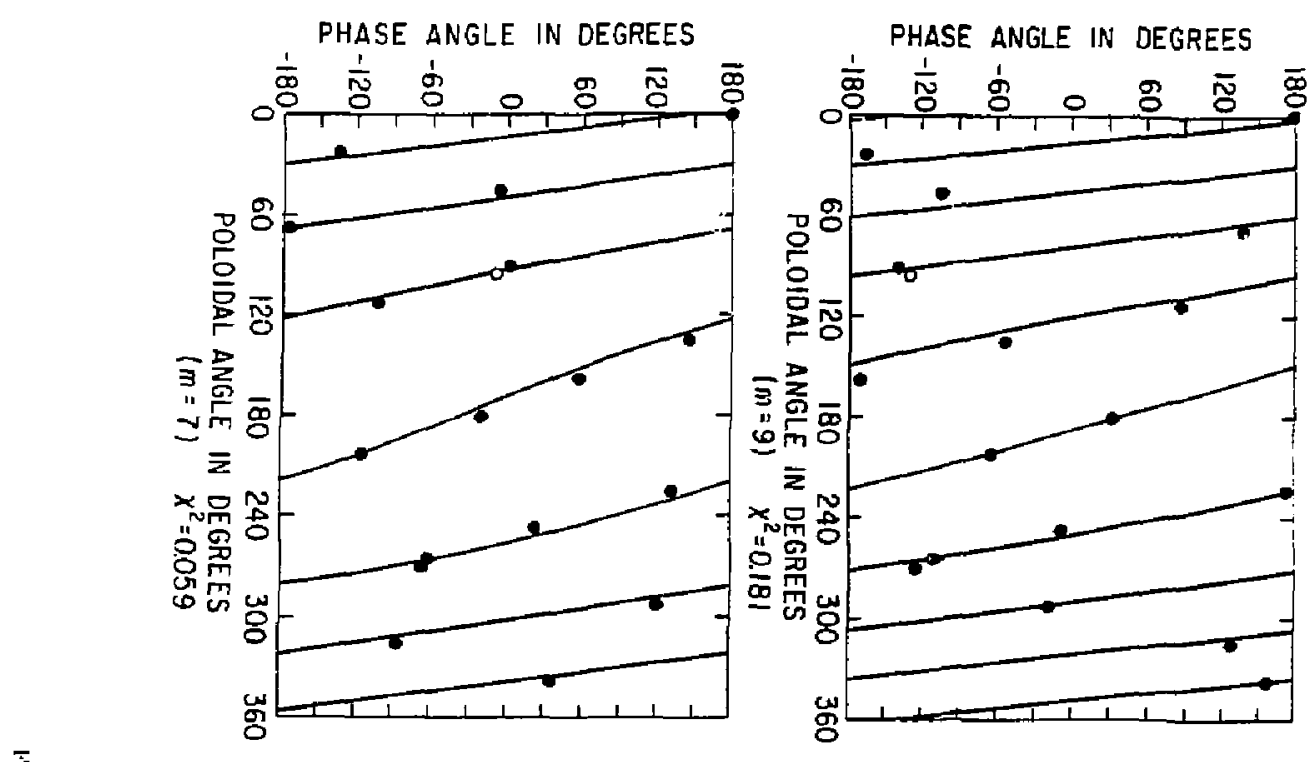

$\Xi$

PHASE ANGLE IN DEGREES

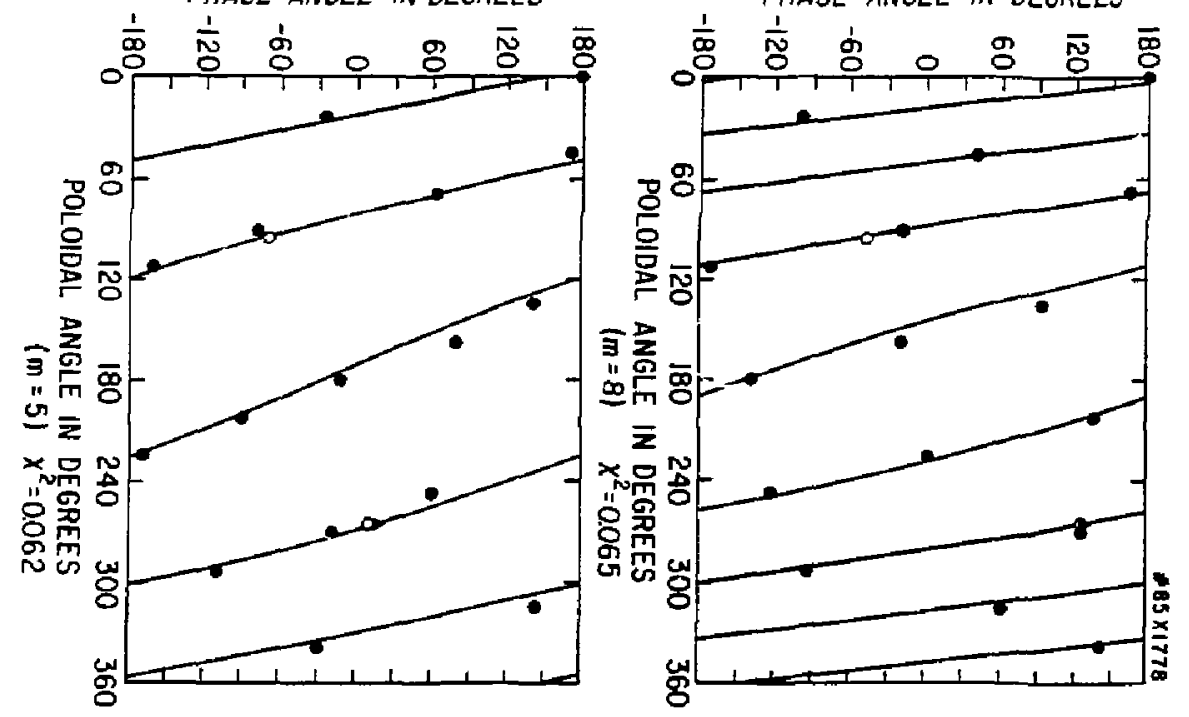




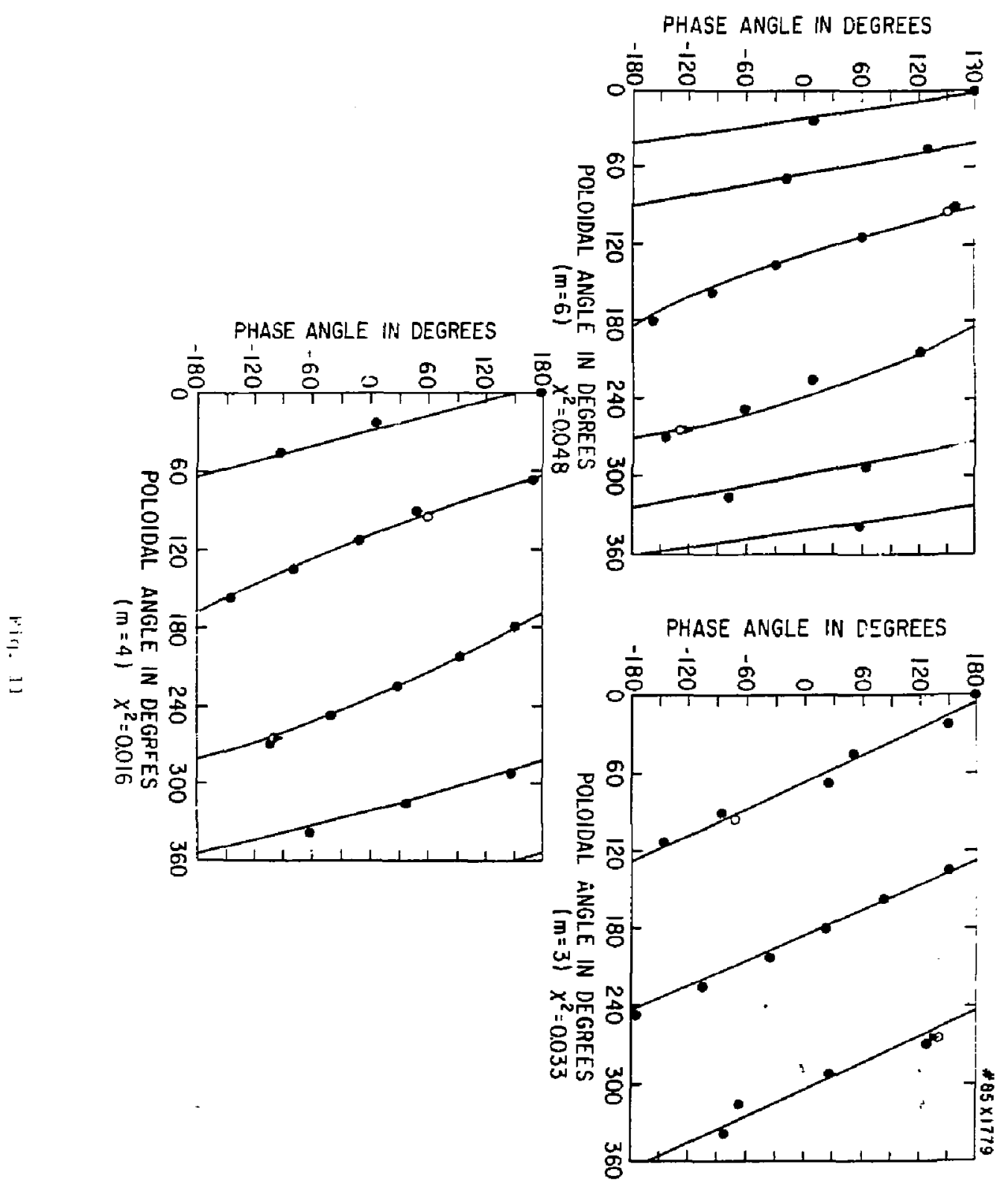



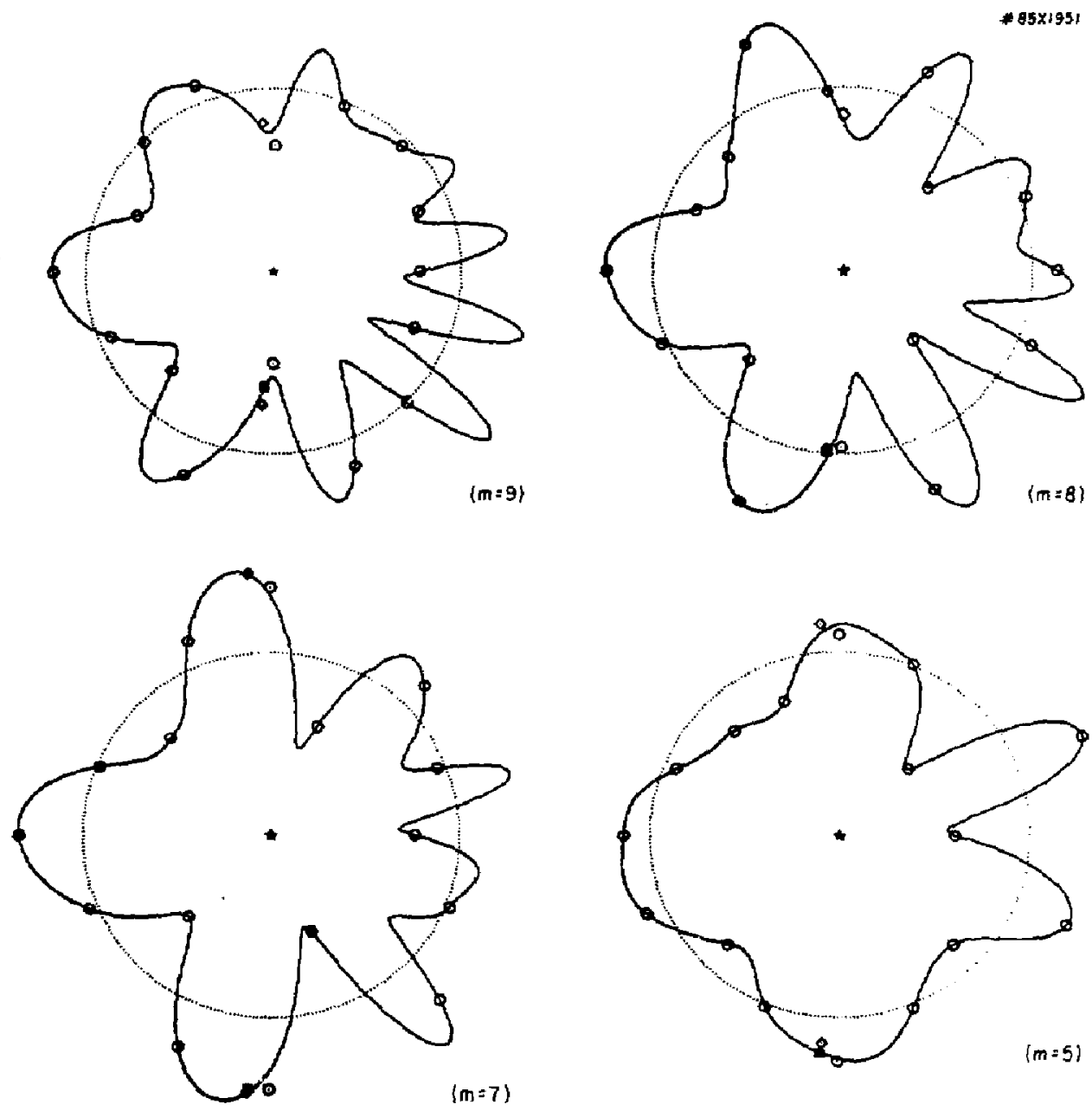

Fig. 12 

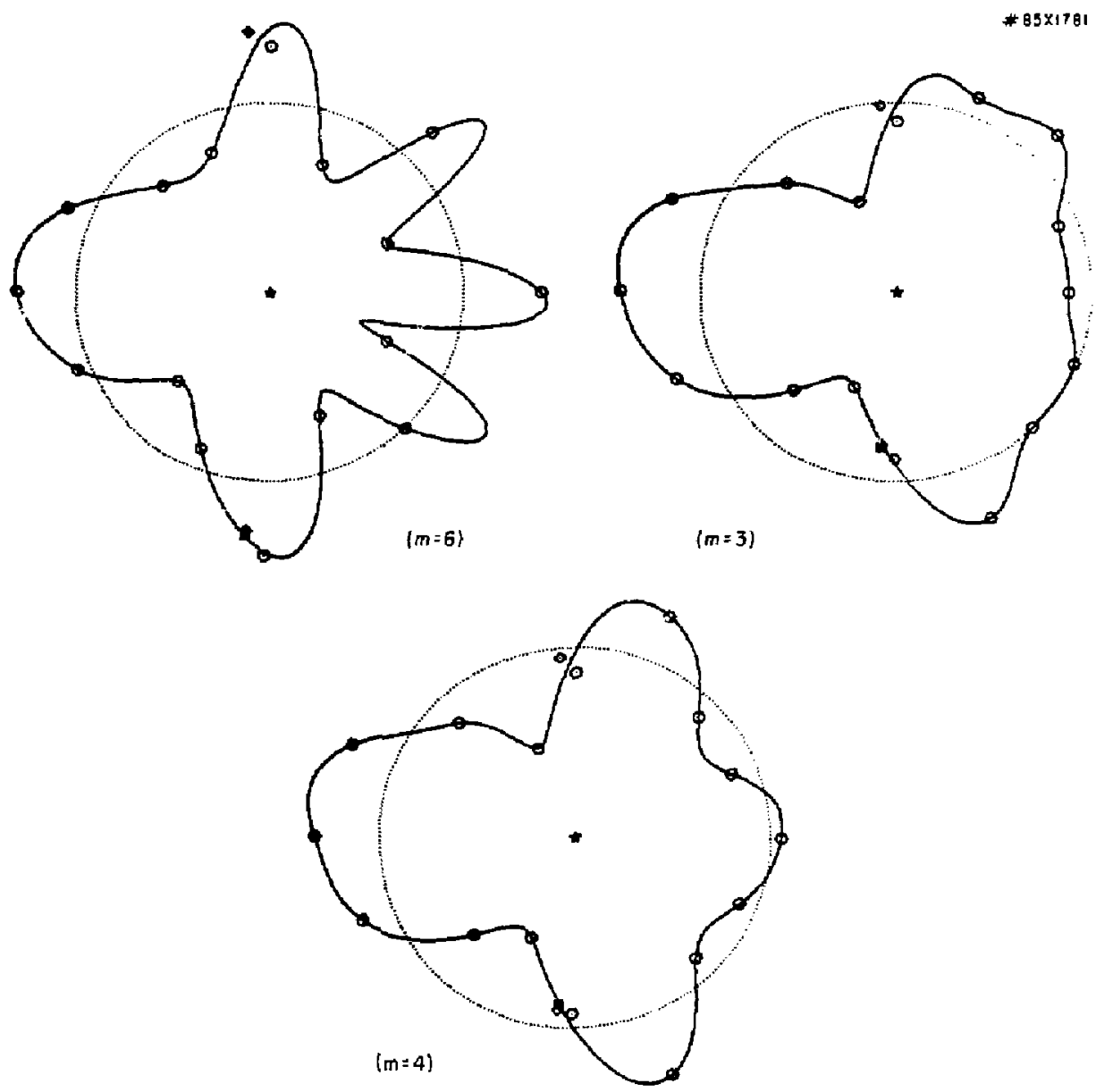

Fig. 13 


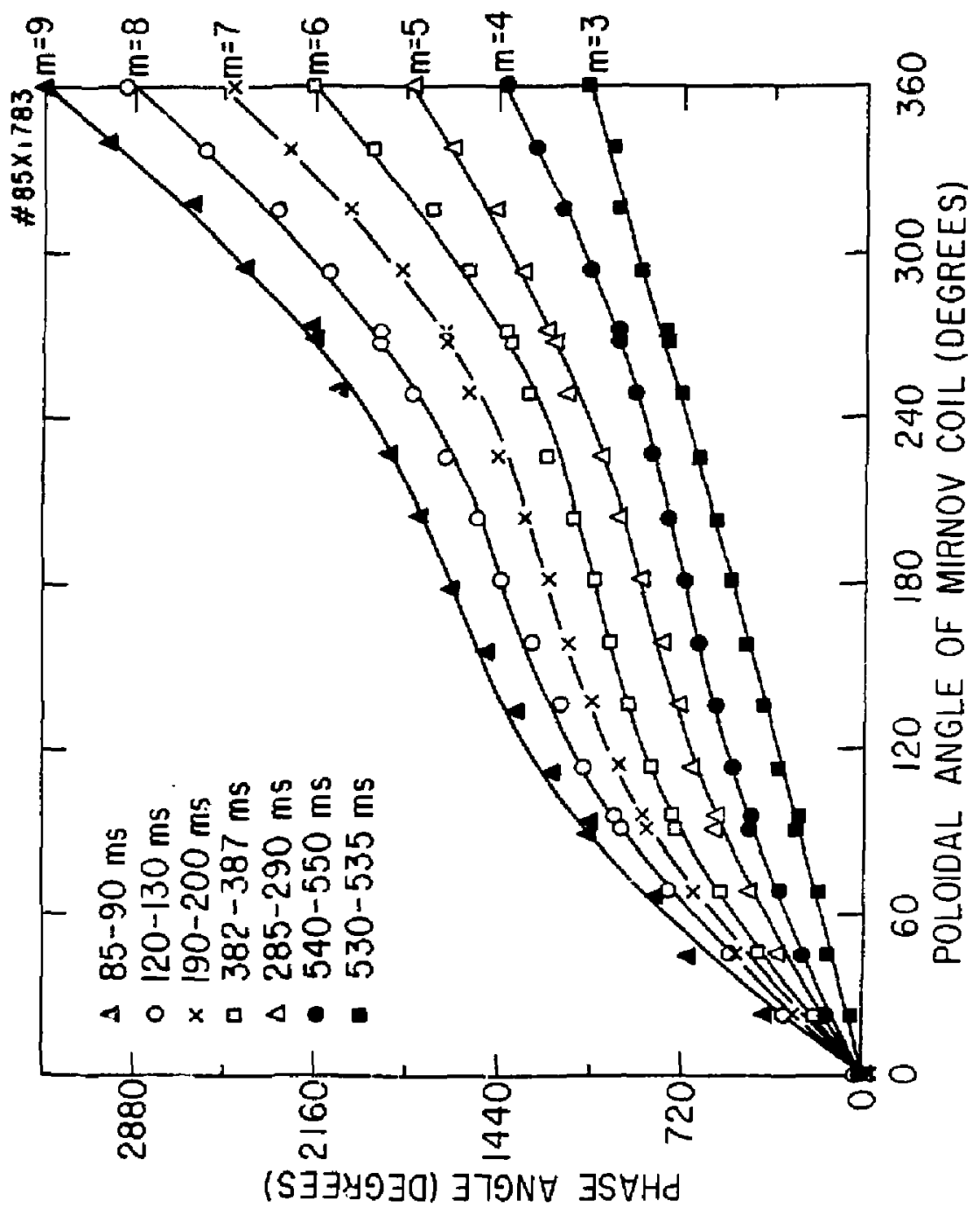



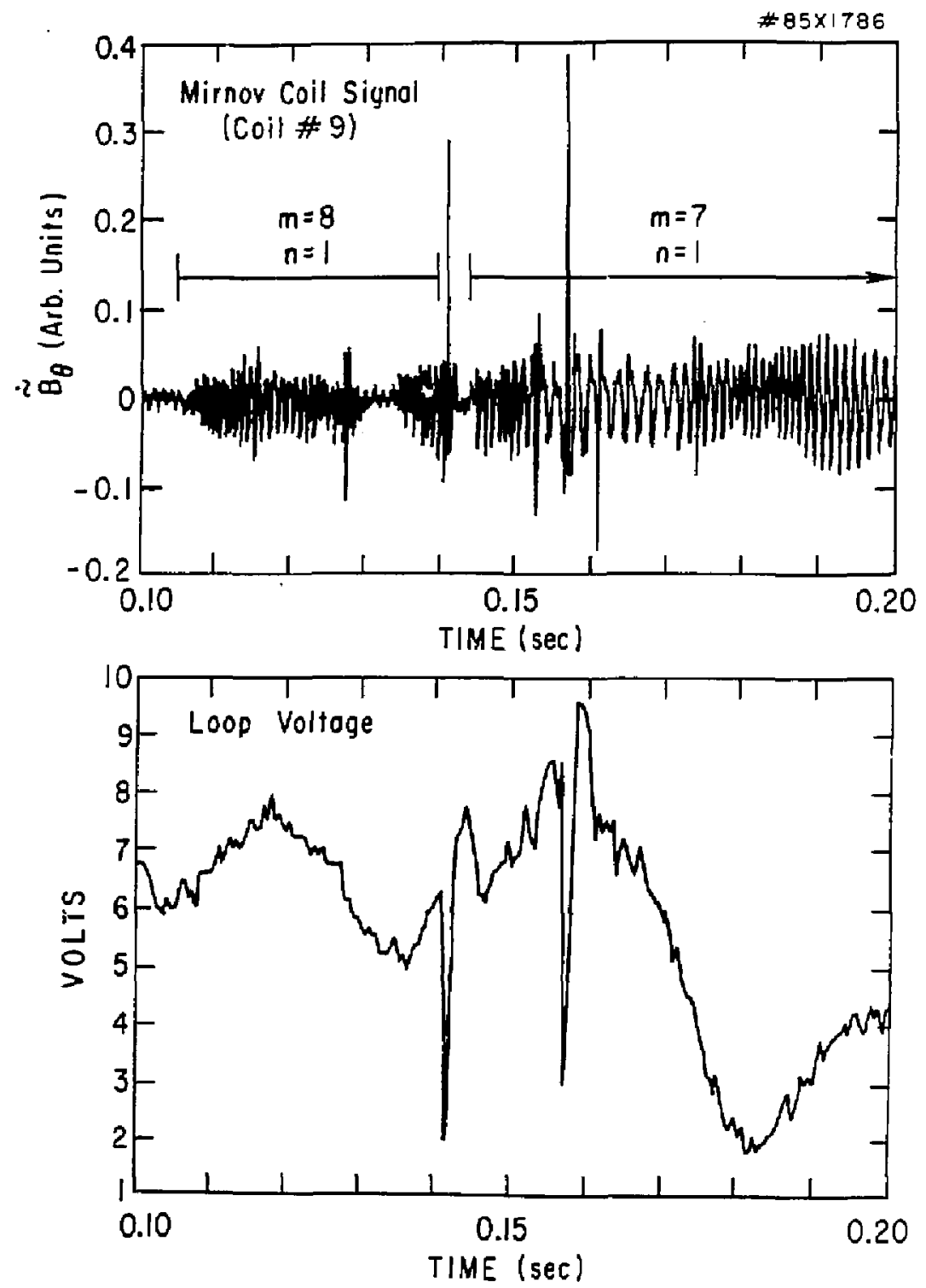

Fig. 15 

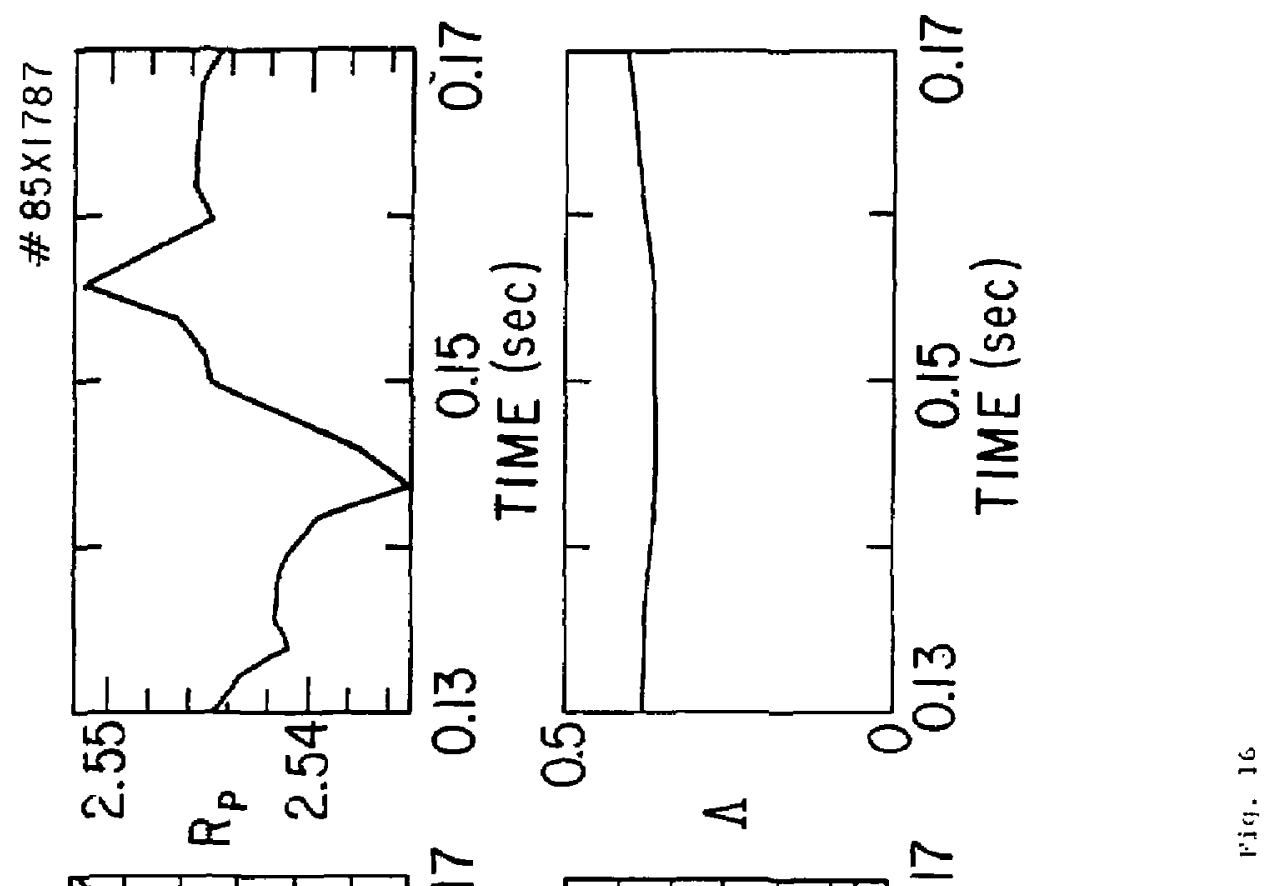

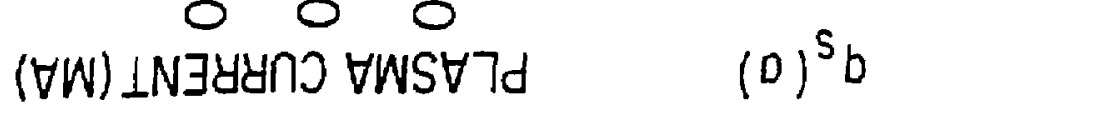



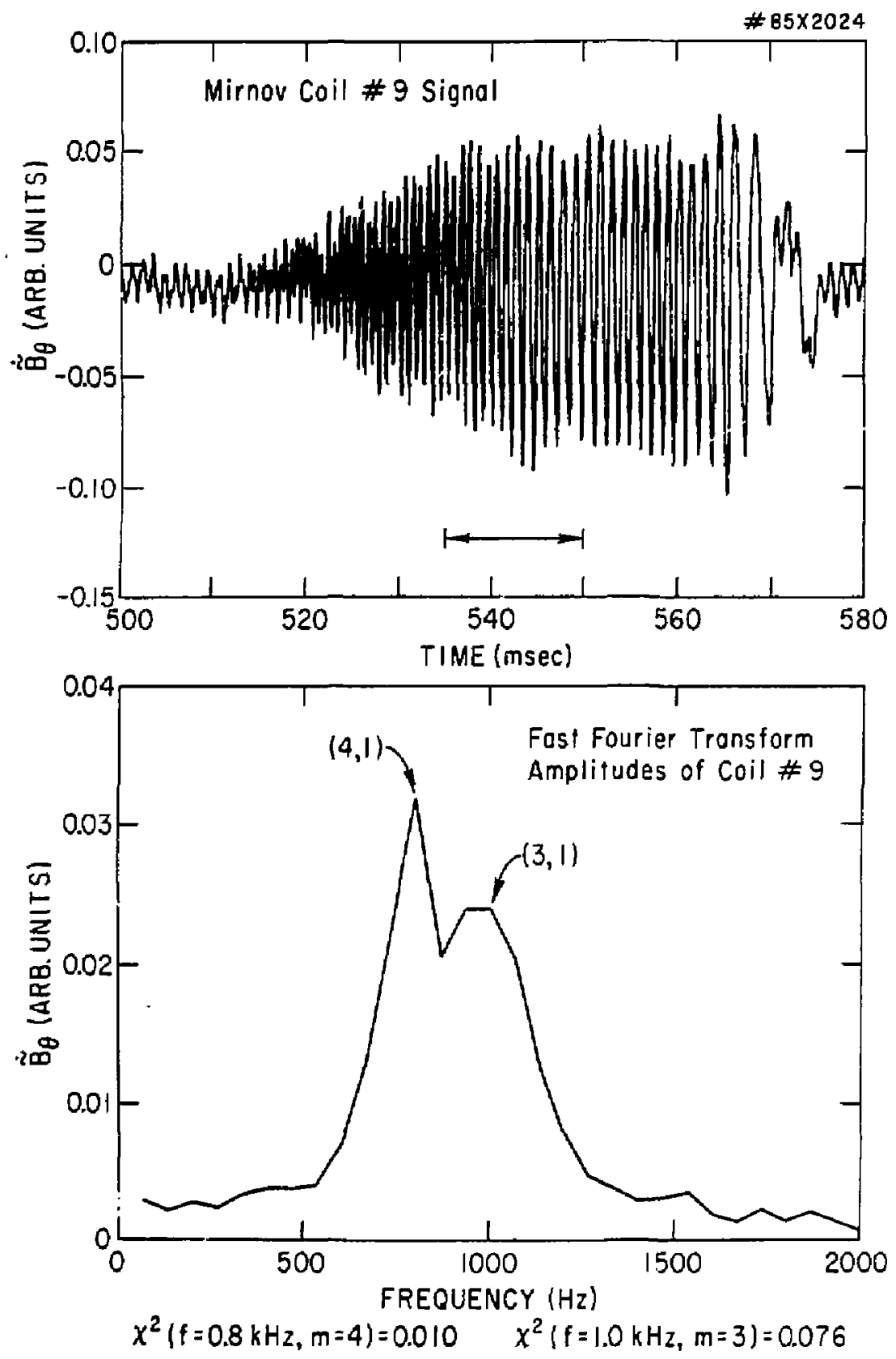

Eig. 17 
\# $85 \times 2025$

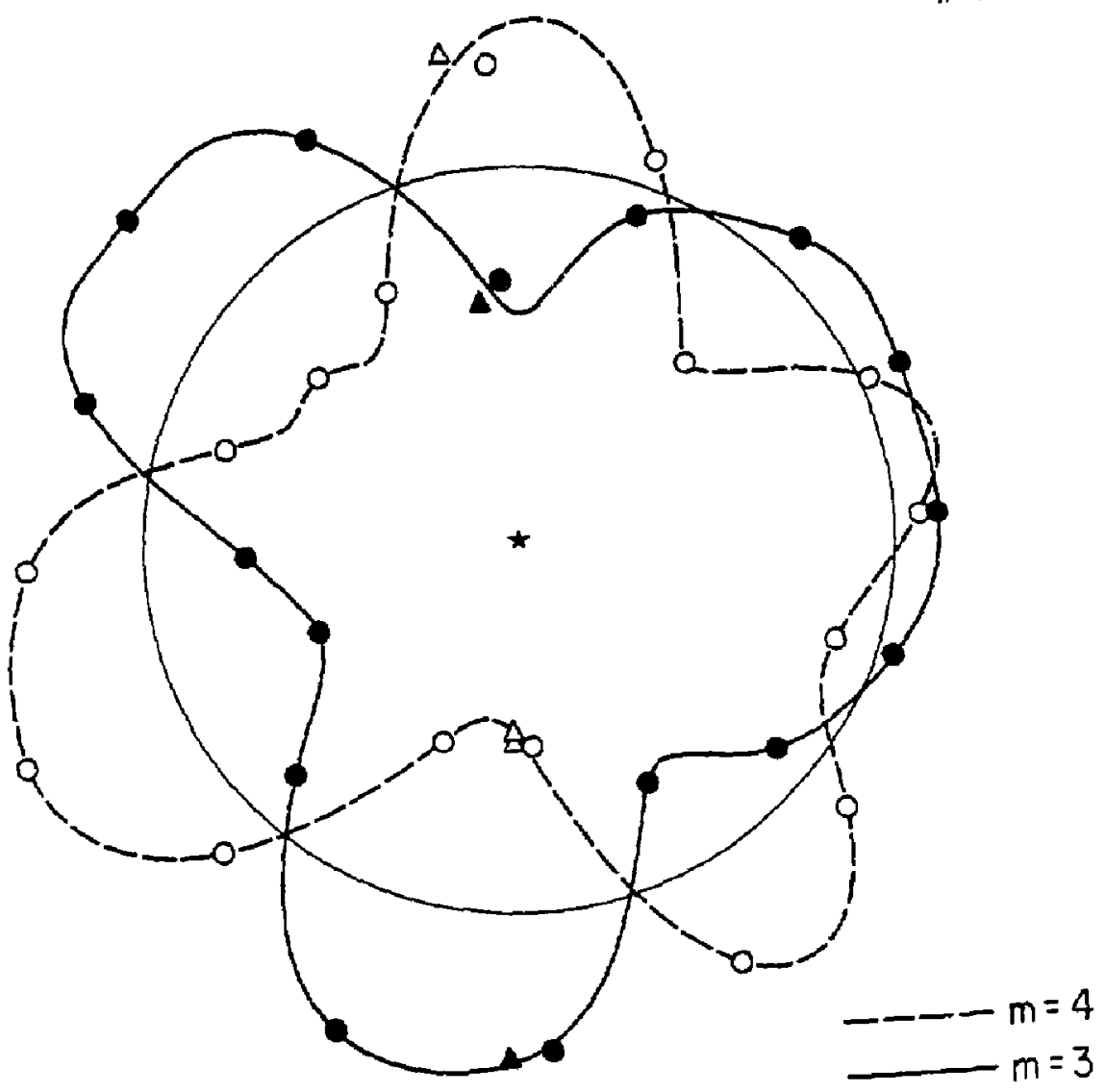

Fig. 18 

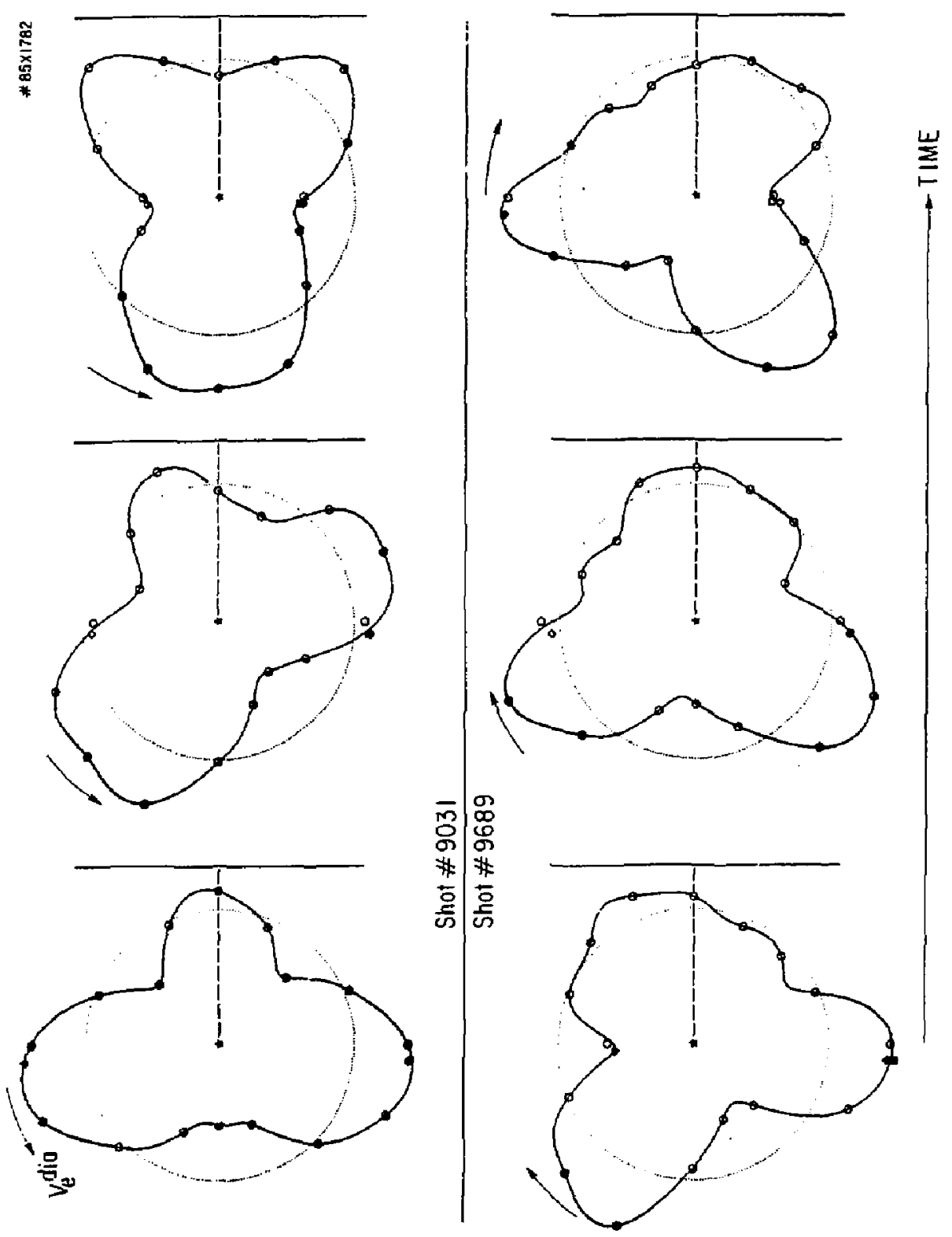

$\Xi$
$\vdots$
$\Xi$ 


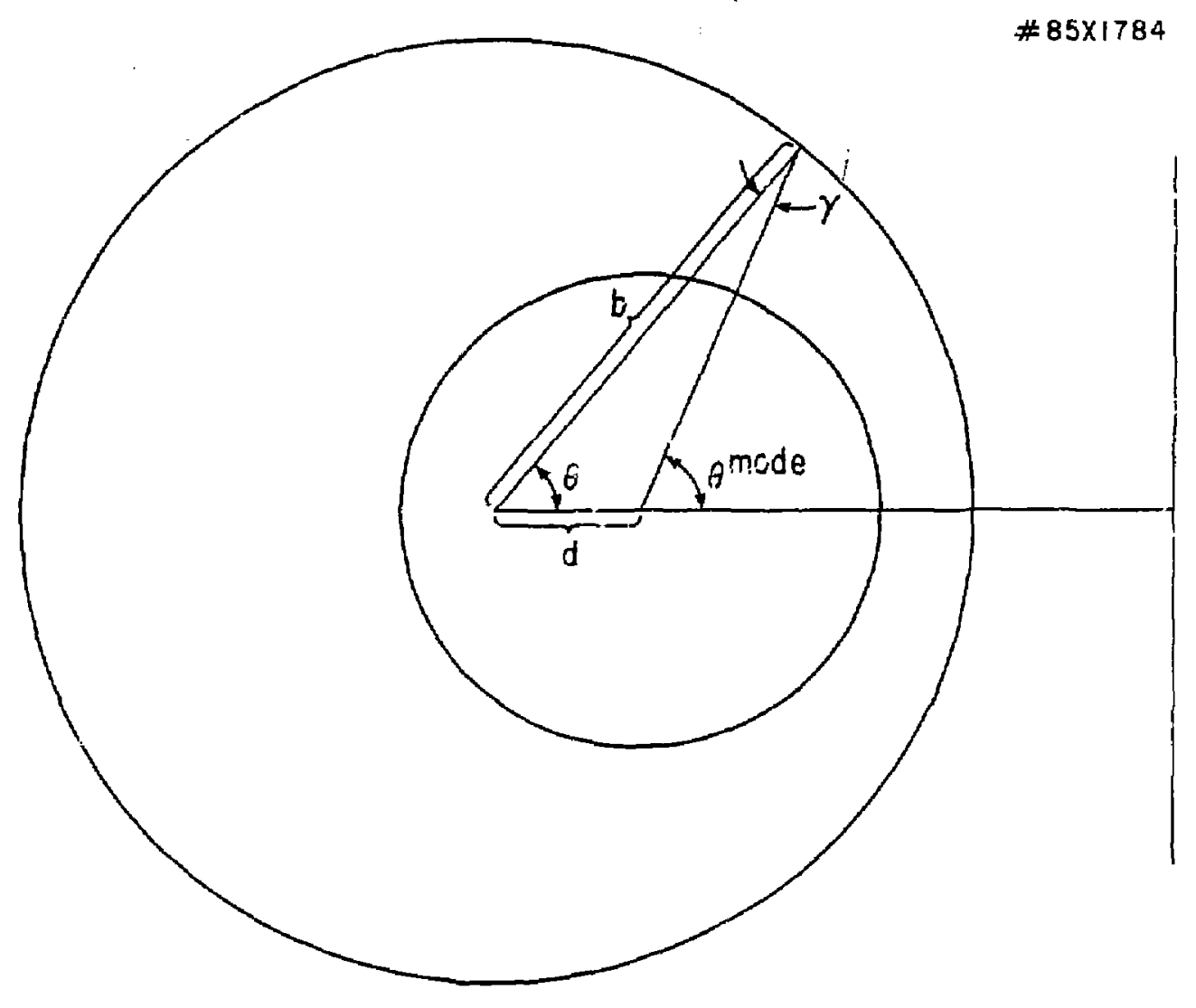

Fig. 20 


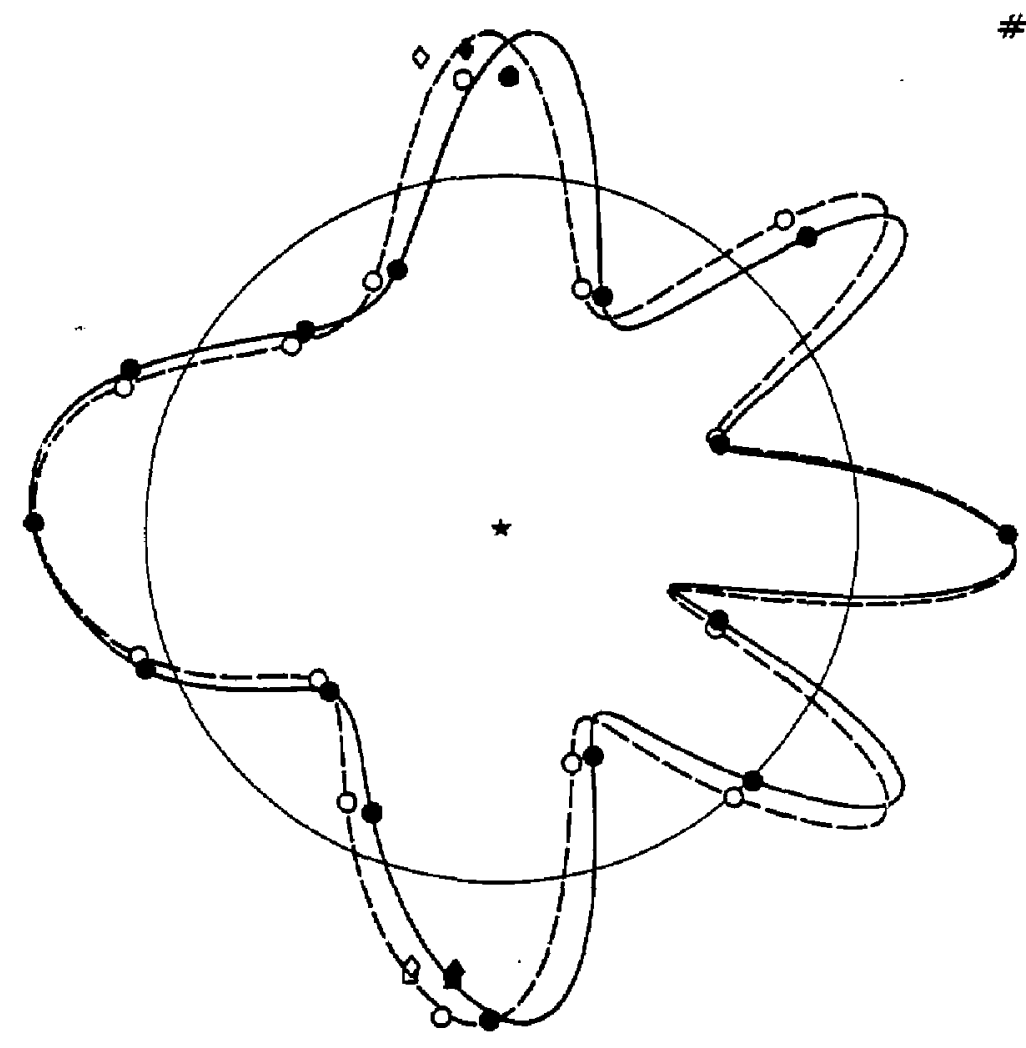

\#85X1785

- Physical Coordinates

--- Mode Coordinates

Fig. 21 
Plasta Res Lab, Austra Nat'1 Univ, AuSTPALTA. D. Jrark J. Paoloni, thiv of Wllongong, Wustrar, th ProE. I.R. Jones, Flinders thiv., ALstRAtIA PToE. Mof. Bcennan, Miv Syand, MUSIPALIA Prot. F. Cap, Irat Theo Foys, ALSTRIA Prof. Fraik verheest, Inst theoritische, BexGmM Dr. D. Palumbo, Dg XIX Eusion Prog, BFGMM Feole Royale Militaice, Lab de Etrys Plagmes, BESTM Dr. P.H. Sakanaka, Univ Fotadial, ERATIL Dr. C.R. Janes, Oniv of albetar CNam Prof. J. Tetchmem, Univ of Mantreal, CANDA DT. H.M. Skargard, utiv of saskatchesan, Cavar Erof. S.R. Sreenivasan, University of Calgary, CANDA prof. Hudor w. Johnston, RPS-Bnergie, CANphs Tr. Hannes Eartian, Ukiv Aritish columbia, OATRA DE. M.P. Cechynsiki, MPB Technologies, Inc. CANPDA Chalk River, Mucl Lab, Cakma

Thengen Li, sinst Physios, CHan

Eibrary, Ising the Universtty, Crmp Tibrarian, Instiute of physics, CIINA Inst Dlasma thys, Acadenia SInica, omp or. Peter Likac, Kcmenskeho Univ, CUDTOSTOAMIA The istbearian, Cuthon Laboratcry, sxzaND Prof. Schatuman, observatoine de Nice, FRAMCE J. Radet, CDS-IXS, ERANC: AM Dupas Iibrary, AM Dupas Lihtary, ERANCE Dr. Tor thal, Acadeny Bibltographic, Haxg kang Preprint Libtary, Cent kes Inst Etrys, Havapt DF. 5,K. Trehan, Panjab Jnivergity, IDDA Dr. Indra than Lal Das, Banaras Hind Chiv, INDIA Ox. In K. Chayda, South Gujarat Univ, INDIA Dr. I.K. Ohlajlani, vikran Univ. IrDIA DF. B. Dasqupo, Stha Inst, IIDIA Dr. P. Kar, Physical Research Lah, DDIA Dx. Fhillip Rosenan, Israel Inst Tech, ISRARt Prof. S, Duperman, TUl Autv thiversity, ISFAES Pxof. G. Rostagni, Univ Di Padovm, IIALY Librarian, Int'l otr Theo Phys, IIfLY

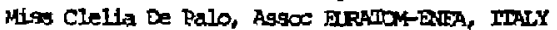
Bihitoteca, del ORR ERATOM, IILLY

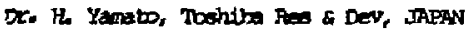
Direc, Dept. Lg, Tokanak Dev, JAerI, JPAN prof. Nohisyiti Inoue, Uhiversity of Tokyo, JAPAN Fesearch Info Center, Magora university, JAPAN prof. Kyoji Nishikaw, thiv of Hiroshima, JAFaN ProE. Sigens WOCi, JAERI, JMPAN isbrary, Kyoto Untuersity, JAPAN PIPE. Iofiro Kawakani, Nihon Univ, JARAs Prof. Satodhi Itoh, Kyught thiversity, JAPAy DE. D.I. Moi, Adv. Inst 5ci \& Itoch, KORER Tech ItFo Divigian, KAERT, KOREA ziblifotheek, Fon-Inst Voor Plagma, NERHERLAND
Prof. B.S. ItIey, Unimersity of waikatos NES ZEALAND Frof. J.A.C. Cabral, Inst superior tecn, poriugat Dr. Octavian Petrus, ALI aJzA University, ROManIA Prof. M.A. Bellbery, University of Natal, SO ARteA Dr. Jchan de villiers, Plasma Physics, Nucor, SO AFRICh Eusion Div, Library, JDS, SPADS Prof. Hans whelison Ghalmen Univ Tech, SAEDN Dr. Lemart Stenflo, University of UMFA, SWFDeN Inbrary, Royal Inst iech, SWEDSN

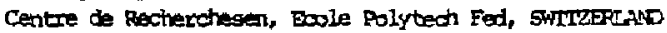
Dr. V.T. Toldk, Kharkov Thys Tech Ins, LSSR Dx. D.D. Ryutov, Siberian Acad Sco, USSR. Dr. G.A. Elisev, Kurctiatov Institute, ISSS: Dr. V.A. Glukhikh, Inst Electro-Physical, USSR Institate Cen Fhysics, USSR ProE. T.J.Me Boyl, Univ College N kales, WaLES Dr. K. Schindler, buhe Oniversitat, w. GERMANy Muclear Res Estab, Julich Ltd, W. GERANY Librarian. Max-Planck Instibit, w. GerMary Bibliothek, Inst Plasmaforsctumg, W. GBRMANY Prof. R.K. Janev, Inst Phys, YuoOsLavIA 